\title{
Searching for Secluded Dark Matter via Direct Detection of Recoiling Nuclei as well as Low Energy Electrons
}

\author{
A. Dedes ${ }^{a}$, I. Giomataris ${ }^{b}$, K. Suxho ${ }^{a}$ and J. D. Vergados ${ }^{a}$ \\ ${ }^{a}$ Division of Theoretical Physics, University of Ioannina, GR 45110, Greece \\ ${ }^{b}$ CEA, Saclay, IRFU, Gif-sur-Yvette, Cedex, France
}

November 3, 2018

\begin{abstract}
Motivated by recent cosmic ray experimental results there has been a proposition for a scenario where a secluded dark matter particle annihilates, primarily, into Standard Model leptons through a low mass mediator particle. We consider several varieties of this scenario depending on the type of mixing among gauge bosons and we study the implications in novel direct dark matter experiments for detecting low energy recoiling electrons. We find significant event rates and time modulation effects, especially in the case where the mediator is massless, that may be complementary to those from recoiling nuclei.
\end{abstract}

\section{Introduction}

The analysis of the positrons excess (vs electrons) seen in cosmic ray spectra from PAMELA $[1,2]$ in the energy region above $10 \mathrm{GeV}$ confirming previous results from HEAT [3,4] and AMS-01 [5] experiments together with very recent results from FERMI [6] and HESS [7] collaborations seems to suggest the presence of a WIMP that annihilates into leptons without any indication of annihilation into $(p, \bar{p})$ pairs or other hadrons (see Refs. $[8,9]$ for relevant analysis). This is also reinforced by ATIC [10] experiment which reports excess of electron plus positron cosmic ray events in the energy region $300 \lesssim E \lesssim 800 \mathrm{GeV}$ and also by signals from WMAP and EGRET [11-13] experiments. These phenomena can be explained by a scenario, originally proposed in ref. [14] - a subset of the so called secluded Dark Matter scenarios [15] - involving a new gauge boson $X_{\mu} 11$, which couples to Standard Model (SM) particles and the WIMP through kinetic vector boson mixing with the following properties [17] :

$$
2 m_{e} \lesssim m_{X} \lesssim m_{\chi} \beta \lesssim m_{\chi} \alpha_{\mathrm{DM}}
$$

where $m_{\chi} \beta$ is a typical non-relativistic WIMP momentum and velocity $\beta \sim 10^{-3}$ inside the galactic halo and $\alpha_{\mathrm{DM}}$ is the dark matter coupling. It has been shown that if eq. (1.1) is satisfied then dark matter annihilation inside the halo to leptons is enhanced by a Sommerfeld factor of $O\left(\alpha_{\mathrm{DM}} / \beta\right)[18]$ while annihilation to protons is simply kinematically forbidden. A typical range of parameters that are going to be exploited in our analysis and satisfy eq. (1.1) are : $m_{X}=0.1-1 \mathrm{GeV}, m_{\chi}=0.1-1$

\footnotetext{
${ }^{1}$ In earlier models [16] of secluded dark matter, WIMPs could be annihilated into new light scalar and gauge bosons.
} 
$\mathrm{TeV}$ and $\alpha_{\mathrm{DM}}=\alpha_{\mathrm{em}}$. The new force mediated by the $\mathrm{X}$-boson is a long range force indeed. We must note here that there is a choice of another viable possibility with an even lighter mediator in $\mathrm{MeV}$ range that has been studied in ref. [19]. Our results for detecting low energy electrons are even more pronounced in this case.

There is also a possibility for the gauge boson mediator $X_{\mu}$ to couple to the SM gauge bosons through a mass mixing matrix in a generalized gauge invariant way. These models are frequently called Stückelberg models [20,21] and are denoted as model type II in our classification. A characteristic of these models is that the electromagnetic current couples to the dark sector through a massless pole identified as the physical photon. As we shall see, this results in considerable and comparable rates in both nucleon or electron recoiling experiments.

Alternatively, it could be that there is a symmetry that renders dark matter particles leptophylic [22-26]. This symmetry is spontaneously broken resulting in a massive gauge boson $X_{\mu}$ that couples directly to both leptons and WIMP at tree level. Again Sommerfeld enhancement dictates the mass of the $X$-boson to be in the $\mathrm{GeV}$ (or sub $\mathrm{GeV}$ ) range. This is the model III that we consider in Chapter 2.

Within the three model categories mentioned above we want :

1. to study the implications of this new force carrier on both traditional nucleon recoil, and untraditional electron recoil direct dark matter searches, and,

2. to suggest new dark matter experiments involving the detection of electrons scattered by this carrier providing a direct link to the recently observed cosmic ray anomalous electron/positron events.

So far there is a dedicated analysis for electron recoils in DAMA experiment [27] with energies approximately $5 \mathrm{KeV}$. Our analysis investigates recoiling electrons with energies as low as 10 $\mathrm{eV}$, and suggests an experimental method on how to reach such low energies. It is therefore complementary to the analysis of Ref. [27].

The structure of this article is as follows : In section 2 we present a field theory setup which helps to categorize three representative model examples that have recently been studied in detail. In section 3, we present event rate predictions for conventional nucleon recoil detection for the models studied. In section 4 , we deal with the not so familiar methods of electron recoil detection rates together with time modulation effects. We also make a proposition of a prototype experiment to be exploited in discovering low energy electrons ejected from WIMP + atom collisions. In section 5 we present our conclusions.

\section{Theory Setup and Model Categories}

In this section, we formulate the problem of the Standard Model coupled to, for simplicity, an Abelian dark sector with arbitrary kinetic or mass mixing terms allowed by Lorentz, gauge symmetries and renormalizability. Our formulae are then applied in subsequent sections to make predictions for event rates in dark matter detection experiments.

To read out the gauge boson propagators we start by writing the general renormalizable form of the Lagrangian :

$$
\mathscr{L}=-\frac{1}{4} \boldsymbol{\Phi}_{\mu \nu}^{T} \mathcal{K} \boldsymbol{\Phi}^{\mu \nu}+\frac{1}{2} \boldsymbol{\Phi}_{\mu}^{T} \mathcal{M}^{2} \boldsymbol{\Phi}^{\mu}-\frac{1}{2} \partial^{\mu} \boldsymbol{\Phi}_{\mu}^{T} \Xi \partial^{\nu} \boldsymbol{\Phi}_{\nu}+\mathbf{J}_{\mu}^{T} \boldsymbol{\Phi}^{\mu},
$$


where $\boldsymbol{\Phi}_{\mu \nu}=\left(\partial_{\mu} \boldsymbol{\Phi}_{\nu}-\partial_{\nu} \boldsymbol{\Phi}_{\mu}\right)$ is a $N$-column matrix field strength tensor corresponding to a $\mathrm{N}$ column $\boldsymbol{\Phi}_{\mu}$ vector field, " $T$ " denotes the transpose of a matrix, $\mathcal{K}$ and $\mathcal{M}^{2}$ are real and symmetric $N \times N$ matrices with model dependent elements to be specified below and $\Xi$ is the gauge fixing $N \times N$ symmetric matrix necessary to remove unphysical gauge degrees of freedom. Interaction terms are encoded in the last term of eq. (2.2) where an external current $\mathbf{J}_{\mu}$ associated with symmetries, couples to the gauge fields.

One has to notice that elements of the mass matrix $\mathcal{M}^{2}$ should be further restricted by electromagnetic gauge invariance. Phenomenologically speaking, there should always be a pole on the propagator $\left\langle\boldsymbol{\Phi}_{\mu} \boldsymbol{\Phi}_{\nu}\right\rangle$ corresponding to the massless photon i.e., the determinant of the inverse propagator at zero momentum must be exactly zero. Furthermore, without loss of generality, we can always assume that the diagonal elements of $\mathcal{K}$ are normalized to unity.

It is standard textbook exercise to find the Feynman propagator, $\widetilde{\mathcal{D}}_{\mu \nu}(p)$ with momentum $p$, for the gauge field $\boldsymbol{\Phi}^{\mu}$ which in momentum space reads,

$$
i \widetilde{\mathcal{D}}_{\mu \nu}(p)=\left(\mathcal{K} p^{2}-\mathcal{M}^{2}\right)^{-1}\left(g_{\mu \nu}-\frac{p_{\mu} p_{\nu}}{p^{2}}\right)+\left(\Xi p^{2}-\mathcal{M}^{2}\right)^{-1} \frac{p_{\mu} p_{\nu}}{p^{2}} .
$$

At lowest order in $\hbar$, interactions among fields are stored in the action functional

$$
S[\widetilde{\mathbf{J}}]=\frac{1}{2} \int \frac{d^{4} p}{(2 \pi)^{4}} \widetilde{\mathbf{J}}_{\mu}^{T}(p)\left[i \widetilde{\mathcal{D}}^{\mu \nu}(p)\right] \widetilde{\mathbf{J}}_{\nu}(-p),
$$

where $\widetilde{\mathbf{J}}_{\mu}(p)$ is the vector current in momentum space. Eqs. (2.3) and (2.4) are what we actually need to describe observables that arise from mixing dark (or hidden) and visible gauge bosons. As a simple example, consider the electromagnetic and the dark gauge boson current. Then in eq. (2.2), it is $\mathbf{J}_{\mu}^{T}=\left(e J_{\mu}^{\text {e.m }}, g_{X} J_{\mu}^{\text {dark }}\right)^{T}$. It is then clear from eq. (2.4) that interactions between the visible and the dark sector will involve off diagonal elements of the propagator (2.3). Observables, like nucleon recoil event rates can easily be described using the above propagator mixing formalism [28], by simply finding the inverse matrices such in eq. (2.3) for a given model. We remark here that the propagator mixing formalism works equally well in different current basis such as $Q-T_{3}$ or $Y-T_{3}$.

\subsection{Model I : Non-standard Kinetic Mixing $\mathcal{K}$}

Models in this category [14,15] have been recently exploited in ref. [17] as candidates for explaining positron excess in cosmic ray data experiments. In its simplest form, the dark matter particle, $\chi$, is charged under a 'dark' $U(1)_{X}$ and the corresponding 'dark' gauge boson $X_{\mu}$ mixes with the photon $A_{\mu}$ and Z-gauge boson, $Z_{\mu}$. Annihilations of dark matter particles into only SM leptons (and not quarks) are kinematically allowed when the intermediate gauge boson has a mass at the GeV scale.

In notation of ref. [29] and in basis $\left(A_{\mu}, X_{\mu}, Z_{\mu}\right)$ (or else $\left.Q-T_{3}\right)$ our matrices $\mathcal{K}$ and $\mathcal{M}^{2}$ appeared in eq. (2.3), become:

$$
\mathcal{K}=\left(\begin{array}{ccc}
1 & -\epsilon \cos \theta_{W} & 0 \\
-\epsilon \cos \theta_{W} & 1 & \epsilon \sin \theta_{W} \\
0 & \epsilon \sin \theta_{W} & 1
\end{array}\right) \quad, \quad \mathcal{M}^{2}=\left(\begin{array}{ccc}
0 & 0 & 0 \\
0 & m_{X}^{2} & 0 \\
0 & 0 & m_{Z}^{2}
\end{array}\right),
$$

where $m_{X}$ is the mass of the exotic gauge boson, $m_{Z}$ is the mass of Z-boson, $\theta_{W}$ is the weak mixing angle and $\epsilon$ is a small $\left(\approx 10^{-3}\right)$ mixing parameter between $U(1)_{Y}$ and $U(1)_{X}$ field strength 


\section{$\stackrel{i}{i} \operatorname{mon} n_{\nu}^{j}=\tilde{D}_{\mu}^{\prime}(p)$}

Figure 1: Diagramatic form of Feynman propagator appeared in eq. (2.3) between gauge boson "flavours" $i$ and $j$. For explicit expressions in model I see Eqs.(2.6)-(2.11); for model II see eq. (2.14).

tensors. Working in Feynman gauge $\left(\Xi=\mathbf{1}_{3 \times 3}\right)$ and keeping up to $\epsilon^{2}$-terms it is easy to work out the mixed propagators $\widetilde{\mathcal{D}}_{\mu \nu}^{i j}(p)$, depicted in Fig $\mathbb{1}$, between photon, $X$ and $Z$-gauge bosons, labeled $1,2,3$, respectively :

$$
\begin{aligned}
i \widetilde{\mathcal{D}}_{\mu \nu}^{11}(p) & =\frac{g_{\mu \nu}}{p^{2}}+\frac{\epsilon^{2} \cos ^{2} \theta_{W}}{p^{2}-m_{X}^{2}}\left(g_{\mu \nu}-\frac{p_{\mu} p_{\nu}}{p^{2}}\right)+O\left(\epsilon^{3}\right), \\
i \widetilde{\mathcal{D}}_{\mu \nu}^{12}(p) & =\frac{\epsilon \cos \theta_{W}}{p^{2}-m_{X}^{2}}\left(g_{\mu \nu}-\frac{p_{\mu} p_{\nu}}{p^{2}}\right)+O\left(\epsilon^{3}\right), \\
i \widetilde{\mathcal{D}}_{\mu \nu}^{13}(p) & =-\frac{\epsilon^{2} p^{2} \cos \theta_{W} \sin \theta_{W}}{\left(p^{2}-m_{X}^{2}\right)\left(p^{2}-m_{Z}^{2}\right)}\left(g_{\mu \nu}-\frac{p_{\mu} p_{\nu}}{p^{2}}\right)+O\left(\epsilon^{3}\right), \\
i \widetilde{\mathcal{D}}_{\mu \nu}^{22}(p) & =\frac{g_{\mu \nu}}{p^{2}-m_{X}^{2}}+\frac{\epsilon^{2} p^{2}\left(p^{2}-\cos ^{2} \theta_{W} m_{Z}^{2}\right)}{\left(p^{2}-m_{X}^{2}\right)^{2}\left(p^{2}-m_{Z}^{2}\right)}\left(g_{\mu \nu}-\frac{p_{\mu} p_{\nu}}{p^{2}}\right)+O\left(\epsilon^{3}\right), \\
i \widetilde{\mathcal{D}}_{\mu \nu}^{23}(p) & =-\frac{\epsilon p^{2} \sin \theta_{W}}{\left(p^{2}-m_{X}^{2}\right)\left(p^{2}-m_{Z}^{2}\right)}\left(g_{\mu \nu}-\frac{p_{\mu} p_{\nu}}{p^{2}}\right)+O\left(\epsilon^{3}\right), \\
i \widetilde{\mathcal{D}}_{\mu \nu}^{33}(p) & =\frac{g_{\mu \nu}}{p^{2}-m_{Z}^{2}}+\frac{\epsilon^{2} p^{4} \sin ^{2} \theta_{W}}{\left(p^{2}-m_{X}^{2}\right)\left(p^{2}-m_{Z}^{2}\right)^{2}}\left(g_{\mu \nu}-\frac{p_{\mu} p_{\nu}}{p^{2}}\right)+O\left(\epsilon^{3}\right) .
\end{aligned}
$$

Some remarks are in order : i) among the three physical masses only $m_{X}^{2}$ mass is shifted by an amount of $m_{X}^{2} \epsilon^{2}$ that we ignore ii) gauge invariance for the off diagonal propagator terms is preserved as should be the case. As far as the effective action eq. (2.4) is concerned, additional statements are in order:

- The single pole $\left[1 / p^{2}\right]$ appears only in $J_{\text {e.m }} \cdot J_{\text {e.m }}$ exchange as usual in the SM.

- A pole $\left[1 /\left(p^{2}-m_{X}^{2}\right)\right]$ for the exotic boson $X_{\mu}$ appears, apart from $J_{X} \cdot J_{X}$ exchange, also in $J_{\mathrm{em}} \cdot J_{X}$ exchange at $O(\epsilon)$.

- There is exchange of current $J_{X} \cdot J_{Z}$ i.e., neutrinos and dark matter particles, through a double pole of $X$ and $Z$ at order $\epsilon$.

- There is exchange of $J_{\mathrm{em}} \cdot J_{Z}$ at order $\epsilon^{2}$ via double pole of $X$ and $Z$

The $\epsilon \approx 10^{-3}$-term in the kinetic mixing can naturally arise as a result of mixing two $U(1)$ 's at high energies - a mechanism that it was first proposed in Ref. [14]. Furthermore, $X$-boson contributions 
to the muon anomalous magnetic moment relative to the SM expectation, $\Delta \alpha_{\mu}=\alpha_{\mu}^{\exp }-\alpha_{\mu}^{\mathrm{SM}}=$ $(290 \pm 90) \times 10^{-11}[30]$, are easily found using eq. (2.6) to be

$$
\Delta \alpha_{\mu}=\frac{\alpha_{\mathrm{em}}}{3 \pi} \epsilon^{2} \cos ^{2} \theta_{W}\left(\frac{m_{\mu}}{m_{X}}\right)^{2}, \quad \text { for } \quad \frac{m_{\mu}}{m_{X}} \ll 1 .
$$

This requires $\epsilon \lesssim 3 \times 10^{-2}$ for $m_{X} \simeq 1 \mathrm{GeV}$ where the equality accounts for the $2 \sigma$ upper limit on $\Delta \alpha_{\mu}$. Of course there are many other constraints on the mixing parameter $\epsilon$ from direct or indirect collider searches and we refer the reader to recent work in Refs. [31-34]. For example, as we see from eqs. (2.6), (2.9) and (2.11) corrections to oblique electroweak observables arise at order $\epsilon^{2}$ similar to the case of muon anomalous magnetic moment.

\subsection{Model II : Non-standard Mass Mixing, $\mathcal{M}^{2}$}

Models belonging to this category are usually referred to as Stueckelberg models [20]. A recent account on "Stueckelberg" extensions of the Standard Model can be found in Ref. [35]. Here, it is more convenient to work on $Y-Y_{X}-T_{3}$ basis $\left(B_{\mu}, X_{\mu}, A_{\mu}^{3}\right)$. We now assume that only the matrix $\mathcal{M}^{2}$ is nontrivial,

$$
\mathcal{K}=\left(\begin{array}{ccc}
1 & 0 & 0 \\
0 & 1 & 0 \\
0 & 0 & 1
\end{array}\right), \quad \mathcal{M}^{2}=\left(\begin{array}{ccc}
\frac{1}{4} g_{Y}^{2} v^{2}+m_{Y}^{2} & m_{Y} m_{X} & -\frac{1}{4} g_{Y} g v^{2} \\
m_{Y} m_{X} & m_{X}^{2} & 0 \\
-\frac{1}{4} g_{Y} g v^{2} & 0 & \frac{1}{4} g^{2} v^{2}
\end{array}\right)
$$

where $g_{Y}, g$ are the $U(1)_{Y}, S U(2)_{L}$ gauge couplings respectively, $m_{Y}^{2}$ is a mass term for the hypercharge gauge field $B_{\mu}$ and $v$ is the vacuum expectation value. The form of the upper left $2 \times 2$ $\mathcal{M}^{2}$ matrix guarantees electromagnetic gauge invariance i.e., massless photon. Furthermore, the zero elements (23) and (32) guarantee that neutrinos are not charged under electromagnetism. Demanding that the inverse propagator has poles at the physical masses, $\left.\operatorname{det}\left[p^{2}-\mathcal{M}^{2}\right]\right|_{p^{2}=m_{i}^{2}}=0$ where $m_{i}=0, m_{X}, m_{Z}$, we find that the photon mass is zero to all orders in $m_{Y}$, the dark gauge boson and the Z-boson masses are not altered up to $O\left(m_{Y}^{2}\right)$, and thus $m_{Z}^{2}=\frac{1}{4}\left(g^{2}+g_{Y}^{2}\right) v^{2}+O\left(m_{Y}^{2}\right)$.

Following eq. (2.4) we obtain the following effective action,

$$
\begin{aligned}
S[J] & =\frac{1}{2} \int \frac{d^{4} p}{(2 \pi)^{4}}\left\{\left[e^{2} J_{\mathrm{e} . \mathrm{m}}(p) \cdot J_{\mathrm{e} . \mathrm{m}}(-p)-2 e^{2} \frac{g_{X}}{g_{Y}} \frac{m_{Y}}{m_{X}} J_{\mathrm{e} . \mathrm{m}}(p) \cdot J_{X}(-p)\right] \frac{1}{p^{2}}\right. \\
& +\left[g_{X}^{2} J_{X}(p) \cdot J_{X}(-p)\left(1-\frac{m_{X}^{2}}{m_{Z}^{2}}\right)+2 e^{2} \frac{g_{X}}{g_{Y}} \frac{m_{Y}}{m_{X}} J_{\mathrm{e} . \mathrm{m}}(p) \cdot J_{X}(-p)\right. \\
& \left.-2 g_{Y} g_{X} \frac{m_{X} m_{Y}}{m_{Z}^{2}} J_{X}(p) \cdot J_{Y}(-p)\right] \frac{1}{p^{2}-m_{X}^{2}} \\
& +\left[g^{2} J_{Z}(p) \cdot J_{Z}(-p)+g_{X}^{2} \frac{m_{X}^{2}}{m_{Z}^{2}} J_{X}(p) \cdot J_{X}(-p)\right. \\
& \left.\left.+2 g_{Y} g_{X} \frac{m_{X} m_{Y}}{m_{Z}^{2}} J_{X}(p) \cdot J_{Y}(-p)\right] \frac{1}{p^{2}-m_{Z}^{2}}\right\}+O\left(m_{Y}^{2}\right),
\end{aligned}
$$

where $e \equiv g_{Y} g / \sqrt{g_{Y}^{2}+g^{2}}$ is the electron charge. Furthermore, $J_{\mathrm{e} . \mathrm{m}}(p)=J_{A_{3}}(p)+J_{Y}(p)$ is the momentum space Fourier transform of the electromagnetic current, i.e., $J_{\mathrm{e} . \mathrm{m}}^{\mu}=\sum_{f} Q_{f} \bar{f} \gamma^{\mu} f$ with 
$Q_{f} e$ being the charge of a generic fermion $f$. The dark current $J_{X}$ obtains an analogous formula with obvious replacement of charge $Q_{f} e$ by another (hyper)charge, $Q_{X}$. Of course, if fermions under consideration are Majorana particles then the corresponding current has only axial-vector form. In addition, $J_{Z}$ denotes the Fourier transform of the Standard Model neutral current $J_{Z}^{\mu}=$ $\frac{1}{\cos \theta_{w}}\left(J_{A_{3}}^{\mu}-\sin ^{2} \theta_{w} J_{\mathrm{e} . \mathrm{m}}^{\mu}\right)$ where the electromagnetic current is, as usual in the SM, the sum of the third component of the isospin $J_{A_{3}}^{\mu}$ and hypercharge currents $J_{Y}^{\mu}$.

The physics of eq. (2.14) is now transparent : to order $\mathrm{O}\left(m_{Y}\right)$, there are interactions between the electromagnetic $J_{\text {e.m }}$ and dark current $J_{X}$ mediated by the photon i.e., the dark matter particle is charged, and interactions between the hypercharge $J_{Y}$ and dark current $J_{X}$ mediated by ( $X$ or $Z$ ) gauge bosons, respectively. An estimate of the dominant contribution to $\Delta \alpha_{\mu}$ results in an upper bound $\frac{m_{Y}}{m_{X}} \lesssim 9 \times 10^{-4}$, where a $2 \sigma$ bound on $\Delta \alpha_{\mu}$ is taken from Ref. [30].

\subsection{Model III : Direct coupling, no mixing}

In this model, some of the SM leptons (but not quarks) $\ell_{L}, e_{R}$ and the WIMP particle $\chi$ are coupled directly to the dark gauge boson $X_{\mu}$ in principle with different coupling@2 2 :

$$
J_{X}^{\mu}=g^{\prime} Y^{\prime}\left(e_{L}\right) \bar{\ell}_{L} \gamma^{\mu} \ell_{L}+g^{\prime} Y^{\prime}\left(e_{R}\right) \bar{e}_{R} \gamma^{\mu} e_{R}+g_{X} Y^{\prime}(\chi) \bar{\chi} \gamma^{\mu} \chi,
$$

where $Y^{\prime}\left(e_{L}, e_{R}\right)=(1,-1)$ denotes the particle hypercharge under the new gauge symmetry. As it has been suggested in Ref. $[8,22,23,25,26]$, this could be an anomaly free gauged $U(1)_{L_{e}-L_{\tau}}$. Of course a new Dirac fermion $\chi$ would be playing the role of dark matter particle is also gauged under this symmetry with $Y^{\prime}(\chi)=1$. Because we have already discussed the effects of the kinetic and mass mixing in the previous models, without loss of generality, we assume that these mixing matrices are trivial in this model at tree leve 3 . If $X_{\mu}$ does not couple to the muon then the most important constraint on $\alpha^{\prime}=g^{\prime 2} / 4 \pi$ will arise from the $\nu-e$ scattering at low $q^{2}$ :

$$
\frac{\alpha^{\prime}}{m_{X}^{2}} \lesssim 7 \times 10^{-7}
$$

We shall use this bound when discussing electron recoil detection rates in section 4 as is typically comparable (most of the time better) with other direct experimental bounds arising from LEP or meson factories. If the $X_{\mu}$ vector boson couples to electrons and muons instead then there is a comparable bound to eq. (2.16) from the muon anomalous magnetic moment. Following $\Delta \alpha_{\mu}=\frac{\alpha^{\prime}}{3 \pi} \frac{m_{\mu}^{2}}{m_{X}^{2}}$ for $m_{\mu} \ll m_{X}$, there is a bound

$$
\frac{\alpha^{\prime}}{m_{X}^{2}} \lesssim 4.4 \times 10^{-6}
$$

\section{Conventional WIMP searches}

Conventional DM searches deal with phenomena of WIMPs scattered of a nucleus. The study of the recoil energy spectrum is the primary goal of experiments such as CDMS [36], XENON [37] and DAMA [38]. For models we described in the previous section there are two cases which have been discussed recently in the literature that could explain the recent anomalous cosmic ray events:

\footnotetext{
${ }^{2}$ Various possibilities on how this is realized can be found in Ref. [22].

${ }^{3}$ Of course mixing of the $X_{\mu}$ gauge boson with the $U(1)_{Y}$ is inevitable at one loop. Its magnitude is calculable : $\epsilon \simeq \alpha^{\prime 2} \log \frac{m_{\tau}}{m_{\mu}}=2 \times 10^{-4}$ for $\alpha^{\prime}=\alpha_{\mathrm{em}}$. All the rest will then proceed following eqs. 2.6- 2.11) of model I.
} 


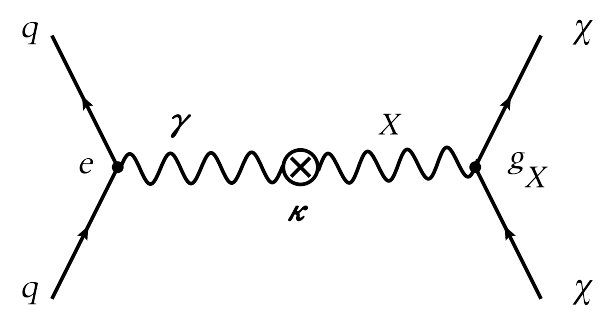

Figure 2: A Feynman diagram leading to the direct interaction of the WIMP $\chi$ to the quarks relevant for direct detection of dark matter. The process is mediated by the physical photon. The cross indicates merely that the exotic gauge boson has a small admixture of the photon. Similarly the WIMP can also couple to electrons.

a) The lightest mediator is massless and

b) the lightest mediator is massive with mass around the proton mass $\left(m_{p}\right)$,

in addition to the assumption that

$$
m_{p} \ll m_{\chi},
$$

where $m_{\chi}$ is the WIMP mass. Only model II belongs to the first category and models I,II belong to the second since by definition, there is no direct coupling of X-boson to quarks in model III [see however footnote 2]. In the following subsections we present the WIMP-nucleon cross section for both cases (a) and (b).

\subsection{Massless Mediator}

The differential WIMP-proton cross section in the rest frame of the initial proton is given by:

$$
d \sigma=\frac{s(\beta)}{\beta} \frac{e^{2}\left(g_{X} \kappa\right)^{2}}{q^{4}} \frac{d^{3} \mathbf{p}^{\prime}}{(2 \pi)^{3}} \frac{d^{3} \mathbf{q}}{(2 \pi)^{3}}(2 \pi)^{3} \delta^{(3)}\left(\mathbf{p}-\mathbf{p}^{\prime}-\mathbf{q}\right)(2 \pi) \delta\left(T-T^{\prime}-T_{q}\right) .
$$

In the above equation $\mathbf{p}^{\prime}, \mathbf{p}$ are the momenta of the initial WIMP and the final WIMP and $\mathbf{q}$ the momentum transfer to the nucleon and $T=p^{2} / 2 m_{\chi}, T^{\prime}=\left(p^{\prime}\right)^{2} / 2 m_{\chi}$ and $T_{q}=q^{2} / 2 m_{p}$, are respectively the corresponding kinetic energies in the non relativistic limit. Furthermore, $\beta$ is the WIMP velocity and $s(\beta)=1$ for a WIMP which is a Dirac fermion, while $s(\beta)=\beta^{2}$ in case it is Majorana one [39] 4 One finds that the momentum transfer and the final nucleon energy are given by:

$$
q=2 \mu_{r} v \xi \approx 2 m_{p} v \xi, T_{q} \approx 2 m_{p} v^{2} \xi^{2},
$$

where $\mu_{r}$ is the WIMP-nucleon reduced mass, $m_{p}$ is the proton mass and $0 \leq \xi \leq 1$ is the cosine of the angle between the incoming WIMP and the outgoing nucleon. Integrating over the momentum of the outgoing WIMP and the magnitude of the momentum of the final hadron as well as the $\phi$-angle one finds :

$$
d \sigma=\frac{s(\beta)}{\beta} \frac{e^{2}\left(g_{X} \kappa\right)^{2}}{2 \pi} \frac{1}{\left(2 m_{p}\right)^{2}} \frac{d \xi}{v^{3} \xi^{3}} .
$$

\footnotetext{
${ }^{4}$ The Majorana fermion does not possess electromagnetic properties. Hence only the $\gamma_{\mu} \gamma_{5}$ of the WIMP $-X$-boson interaction contributes.
} 
The above expression exhibits, of course, the infrared divergence. We will impose a low momentum cut off $E_{\mathrm{th}} / A$ provided by the energy threshold $E_{\mathrm{th}}$, where $\mathrm{A}$ is the mass number of the target, i.e.

$$
\xi_{\text {min }}=\sqrt{\frac{E_{\mathrm{th}}}{\left(2 A m_{p} \beta^{2}\right)}} .
$$

Thus the total cross-section for a Majorana WIMP is given by:

$$
\sigma=\frac{\alpha}{2}\left(g_{X} \kappa\right)^{2} \frac{1}{\left(m_{p}\right)^{2}}\left(\frac{A m_{p}}{E_{\mathrm{th}}}-\frac{m_{p}}{T_{\max }}\right) \approx \frac{\alpha}{2}\left(g_{X} \kappa\right)^{2} \frac{1}{\left(m_{p}\right)^{2}} \frac{A m_{p}}{E_{\mathrm{th}}} .
$$

Eq. (3.23) shows a much stronger dependence of the event rate on the threshold energy $E_{\text {th }}$ due to the adopted cut-off $E_{\text {cut-off }}=E_{\text {th }} / A$. It is interesting to note that this cross section is independent of the WIMP velocity (in the case of a Dirac WIMP the extracted from the data cross section must be multiplied by $\beta^{2}$ ). We distinguish two cases :

1. The case of Majorana WIMP. We find:

$$
\sigma \approx 1.6 \times 10^{-30} \mathrm{~cm}^{2}\left(g_{X} \kappa\right)^{2} \frac{2 A m_{p}}{E_{t h}} .
$$

The direct dark matter experiments have recently set on the coherent nucleon cross section the limits:

- The CDMSII experiment [36]:

The best limit is $6.6 \times 10^{-44} \mathrm{~cm}^{2}$. The extracted value depends, however, on the assumed WIMP mass. So it can vary between $6.6 \times 10^{-44}$ and $6.6 \times 10^{-42} \mathrm{~cm}^{2}$.

- The XENON10 collaboration [37]

They extract $8.8 \times 10^{-44} \mathrm{~cm}^{2}$ and $4.5 \times 10^{-44} \mathrm{~cm}^{2}$ for WIMP masses of 100 and $30 \mathrm{GeV}$ respectively.

For our purposes we will assume that the extracted from the data nucleon cross section is $10^{-7} \mathrm{pb}=10^{-43} \mathrm{~cm}^{2}$. Furthermore we will take as a reference a threshold energy of 5.0 $\mathrm{KeV}$ and examine the sensitivity of our results to the experimental threshold. Using the experimental limit, $\sigma_{p} \leq 1.0 \times 10^{-43} \mathrm{~cm}^{2}$, we can write:

$$
\frac{\text { Rate }(\text { new })}{\text { Rate }(\text { conventional })}=1.6 \times 10^{6} \frac{Z^{2}}{A^{2}}\left(g_{X} \kappa\right)^{2} \frac{A m_{p}}{E_{t h}} .
$$

Note that the coherence factor now is $Z^{2}$, since in the case of the photon only the protons of the target contribute. Adopting a threshold value of $5 \mathrm{KeV}$, we get

$$
\frac{\text { Rate }(\text { new })}{\text { Rate }(\text { conventional })}=3.0 \times 10^{18} \frac{Z^{2}}{A}\left(g_{X} \kappa\right)^{2} .
$$

For the Ge target $(A=73, Z=32)$ we get

$$
\frac{\text { Rate }(\text { new })}{\text { Rate }(\text { conventional })}=4.3 \times 10^{19}\left(g_{X} \kappa\right)^{2}
$$


which leads to the limit:

$$
\left|g_{X} \kappa\right| \leq \sqrt{\frac{1}{0.43 \times 10^{19}}}=1.6 \times 10^{-10} .
$$

From the second term in eq. (2.14) and assuming that $\alpha_{\mathrm{DM}}=g_{X}^{2} / 4 \pi=\alpha_{\mathrm{em}}$ one can easily translate this into bounds on the model II parameters for Majorana WIMP :

$$
Q_{X} \frac{m_{Y}}{m_{X}} \lesssim 0.54 \times 10^{-10}, \quad \text { model II . }
$$

2. The case of a Dirac WIMP. We find:

$$
\sigma \approx \frac{1}{\beta^{2}} \frac{\alpha}{2} \frac{1}{\left(m_{p}\right)^{2}}\left(g_{X} \kappa\right)^{2} \frac{A m_{p}}{E_{t h}} .
$$

If we knew the coupling $\left|g_{X} \kappa\right|$ we could incorporate this into the evaluation of the nuclear cross section, fold it with the velocity distribution and proceed with the evaluation of the event rate. Since, however, we like to constrain the parameter $\left|g_{X} \kappa\right|$ we will employ an average velocity:

$$
\sigma \rightarrow<\sigma>\approx<\frac{1}{\beta^{2}}>\frac{\alpha}{2} \frac{1}{\left(m_{p}\right)^{2}}\left(g_{X} \kappa\right)^{2} \frac{A m_{p}}{E_{t h}} .
$$

But for a Maxwell - Boltzmann distribution i.e., $\quad<\frac{1}{\beta^{2}}>\rightarrow \frac{3}{<\beta^{2}>}$, we obtain the constraint:

$$
\left|g_{X} \kappa\right| \leq 1.6 \times 10^{-10} \frac{\sqrt{<\beta^{2}>}}{\sqrt{3}} \approx 0.8 \times 10^{-13}
$$

from which the bound on model II for $\alpha_{\mathrm{DM}}=\alpha_{\mathrm{em}}$,

$$
Q_{X} \frac{m_{Y}}{m_{X}} \lesssim 0.27 \times 10^{-13}, \quad \text { model II }
$$

is found. As expected the limit is now more stringent than in eq. (3.29).

The results for the Xe target are similar. This bound is by many orders of magnitude stronger than the one obtained from electroweak fits [35] or $(g-2)_{\mu}$ [see discussion towards the end of section (2.2)]. The corresponding bound for Dirac WIMP is about three orders of magnitude more stringent. This means that additional mechanisms should be added in model II (Stückelberg type of Ref. [35] for example) in order to efficiently depleting the WIMP in the early universe (the diagram in Fig. 2 is just the crossing diagram of the annihilation cross section).

Although eq. (3.29) [or eq. (3.33)] provides a very stringent limit, we should not forget that in this case we have a much stronger dependence of the rates on the energy threshold through the need for a low energy cut off on the elementary cross section.

Alternatively we may extract from the data for Xe $(A=131, Z=54)$ an elementary cross section assuming it to be of the form 5 :

$$
\sigma_{N, \chi^{0}}^{S}\left(A, E_{\mathrm{th}}\right)=\sigma_{0} \frac{A}{131} \frac{5 \mathrm{keV}}{E_{\mathrm{th}}},
$$

\footnotetext{
${ }^{5}$ This treatment does not distinguish between a Majorana and a Dirac WIMP.
} 


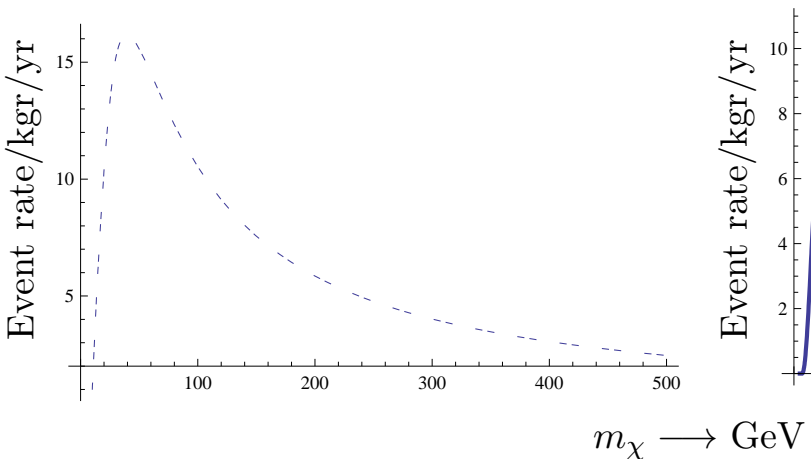

(a)

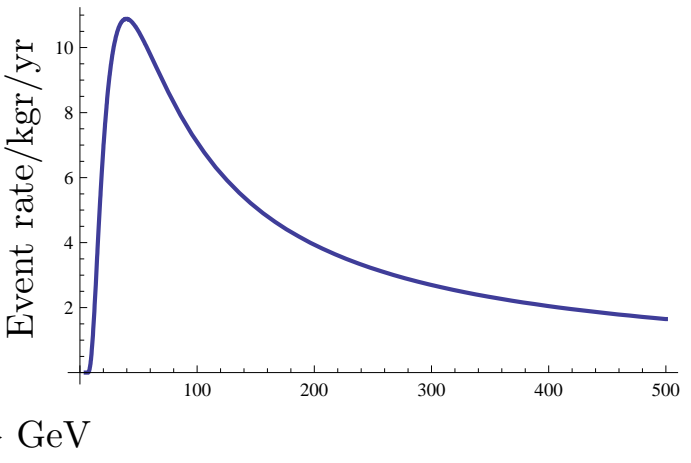

(b)

Figure 3: The total rates for traditional WIMP searches assuming a nucleon cross section $\sigma_{N}=$ $10^{-43} \mathrm{~cm}^{2}$ in (a). The case of the photon mediated process considered in this work is exhibited in (b). Both refer to the case of a heavy target $(A=131)$ and were computed assuming an energy threshold of $5 \mathrm{KeV}$. The results for the Iodine target used by the DAMA experiment are almost identical.

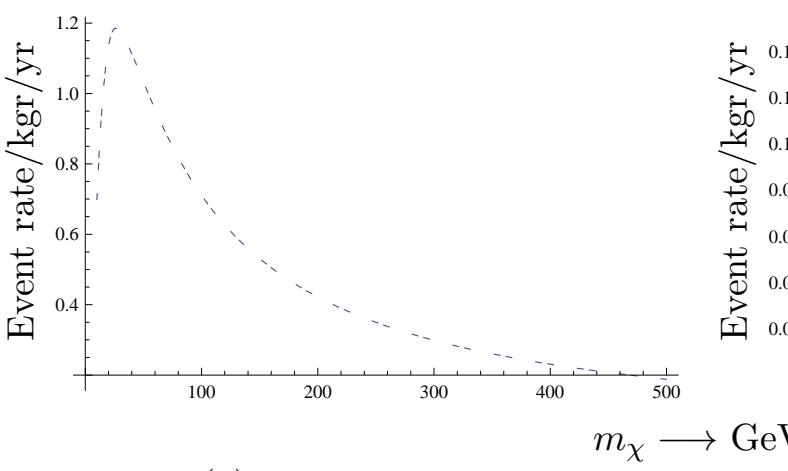

(a)

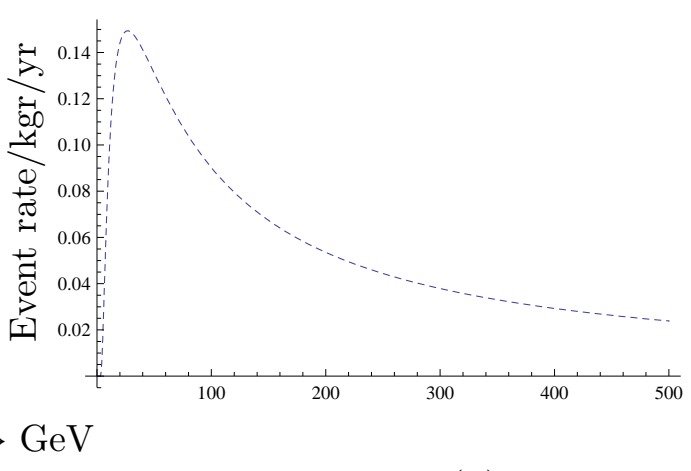

(b)

Figure 4: The same as in Fig. 3 in the case of the light target ${ }^{19} \mathrm{~F}$.

where $\sigma_{0}$ is the elementary cross section obtained in the particle model for a target with nuclear mass number $A$ and threshold energy $E_{\text {th }}$. Then by fitting to the experiment we obtain

$$
(131 / 54)^{2} \sigma_{N, \chi^{0}}^{S}=0.5 \times 10^{-7} \Rightarrow \sigma_{0}=2.9 \times 10^{-7} \mathrm{pb}=2.9 \times 10^{-43} \mathrm{~cm}^{2} .
$$

In spite of the $(Z / A)^{2}$ factor we obtain a smaller value than in the standard experiment. This is due to the small cut off energy $E_{\mathrm{th}} / A$ employed. With the above ingredients the number of events in time $T$ due to the coherent scattering [40], can be cast in the form:

$$
\begin{aligned}
& R \simeq 1.0710^{-5} \times \\
& \quad \frac{T}{1 \mathrm{y}} \frac{\rho(0)}{0.2 \mathrm{GeVcm}^{-3}} \frac{100 \mathrm{GeV}}{m_{\chi^{0}}} \frac{m}{1 \mathrm{~kg}} \frac{\sqrt{\left\langle v^{2}\right\rangle}}{280 \mathrm{~km} \mathrm{~s}^{-1}} \frac{\sigma_{N, \chi}^{S}}{10^{-43} \mathrm{~cm}^{2}} f_{\mathrm{coh}}\left(A, \mu_{r}(A)\right),
\end{aligned}
$$

where the elementary cross section $\sigma_{N, \chi}^{S}$ can be treated as a phenomenological parameter independent of the WIMP mass in units of $10^{-43} \mathrm{~cm}^{2}$. The quantity $f_{\text {coh }}\left(A, \mu_{r}(A)\right)$ can be obtained from 


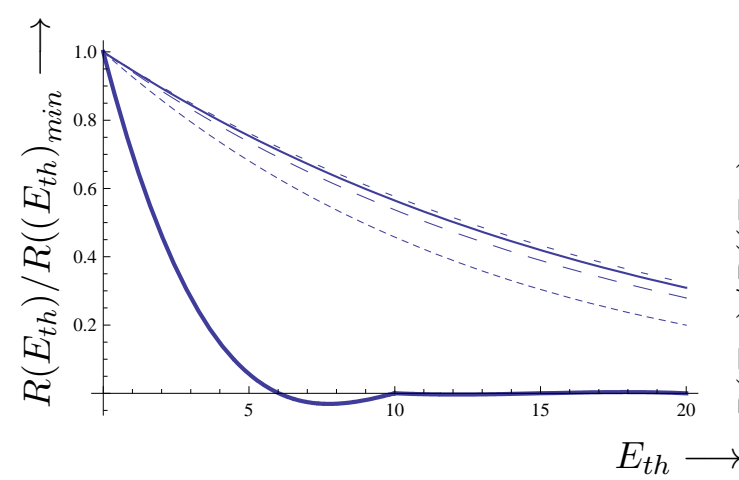

(a)

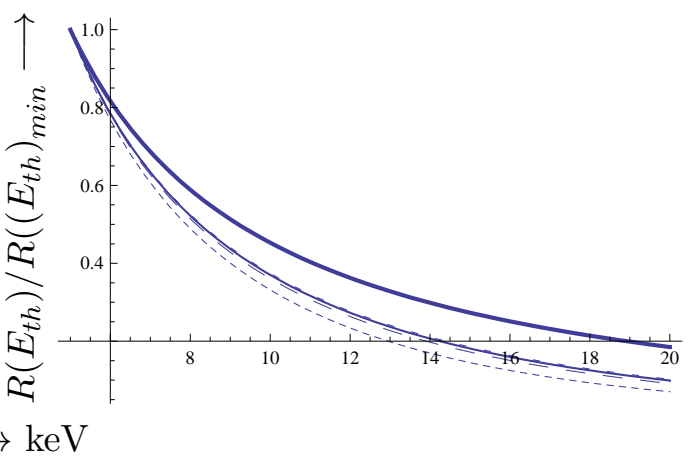

(b)

Figure 5: The quantity $R\left(E_{\mathrm{th}}\right) / R\left(\left(E_{\mathrm{th}}\right)_{\text {min }}\right.$, i.e. the ratio of the event rate at a given threshold divided by that at the lowest threshold considered, as a function of the threshold energy. In (a) as predicted by traditional mechanisms (lowest threshold assumed zero). In (b) as predicted by the present model (now due to the need for a cut off the lowest threshold energy employed was $5 \mathrm{keV}$ ). The thick line, short dash, long dash, fine line and long short dash correspond to WIMP masses 10, 50, 100, 200 and $500 \mathrm{GeV}$ respectively.

the published in Ref. [40] values of $t$ for the standard MB velocity distribution $(n=1)$. For the photon mediated mechanism examined here the above equation must be modified by multiplying $f_{\text {coh }}\left(A, \mu_{r}(A)\right)$ with the factor $Z^{2} / A^{2}$ and employing eq. (3.34) for the elementary cross section (in units of $10^{-43} \mathrm{~cm}^{2}$ ). The event rate per $\mathrm{kg}$ of target per year for the traditional experiments for a heavy isotope like Xe and a light isotope like ${ }^{19} \mathrm{~F}$, as a function of the WIMP mass is exhibited in Figs 3 and 4. On the same plots we show the event rate for the photon mediated process examined in the present work. It is not surprising that the agreement is good since the elementary cross section was fitted to the data. The small difference is understood, since in the extraction of the elementary cross section from the data a zero threshold value was used in the phase space integrals. The event rates are sensitive functions of the threshold energy, $R=R\left(E_{\mathrm{th}}\right)$. In the case of the Xe isotope the ratio $R\left(E_{\mathrm{th}}\right) / R\left(\left(E_{\mathrm{th}}\right)_{\min }\right.$ is exhibited in Fig. 5. The threshold dependence is much more profound in the case of the light WIMP, since, then, the average energy transfered is small. As expected the threshold dependence is more dramatic in the case of the present model (this is a bit obscured in the figure since in this case the graphs are normalized at $5 \mathrm{keV}$ ).

In the case of a Dirac fermion the extracted limit will be smaller, but the traditional calculations are not adequate for the analysis, due to the different velocity dependence of the elementary cross section.

\subsection{Massive Mediator}

In this case the WIMP - nucleon cross section reads :

$$
\begin{aligned}
\sigma & =s(\beta) \frac{16 \pi \alpha_{\mathrm{em}} \kappa^{2} \alpha_{\mathrm{DM}} m_{p}^{2}}{m_{X}^{4}} \\
& =1.2 \times 10^{-30} \mathrm{~cm}^{2} s(\beta) \frac{\alpha}{137^{-1}} \frac{\alpha_{\mathrm{DM}}}{137^{-1}} \kappa^{2}\left(\frac{m_{p}}{m_{X}}\right)^{4},
\end{aligned}
$$


where the cross section refers to Dirac (Majorana) WIMP and $s(\beta)=1\left(\beta^{2}\right)$ respectively. Taking $\beta^{2} \rightarrow<\beta^{2}>\approx 10^{-3}$ we find:

$$
\kappa \lesssim 3 \times 10^{-7}\left(3 \times 10^{-4}\right)
$$

From these we obtain bounds for parameters in models I,II [see eqs. (2.8) and (2.14)] ,

$$
\begin{aligned}
\epsilon & \lesssim 3.0 \times 10^{-7}\left(3.0 \times 10^{-3}\right), & & \text { Model I } \\
Q_{X} \frac{m_{Y}}{m_{X}} & \lesssim 1.6 \times 10^{-6}\left(1.6 \times 10^{-3}\right), & & \text { Model II },
\end{aligned}
$$

where the number in parenthesis corresponds to Majorana WIMP dark matter particle. These limits are less stringent than those obtained in the case of the massless mediator.

In the case of the massive mediator, with the possible exception of the velocity dependence in the case of Majorana WIMP, the cross section behaves as in the standard CDM case, since in this case we do not encounter an energy cutoff. Since, however, we do not know the values of the parameters $\epsilon$ and $\frac{m_{Y}}{m_{X}}$, we cannot make predictions about the event rates. Instead we have used the present experimental limits to constrain these parameters. Thus we saw that the current experimental limits impose the most stringent limits on these parameters. If, on the other hand, we use the previous constrains we can conclude that WIMPs in models I,II scatter off nuclei too many times. These effects should have been seen in experiments [36,37] (or may have already been seen [38]). An exception is a Majorana WIMP candidate in model I which results in current sensitivity event rates.

\section{Unconventional WIMP searches}

\subsection{Cross Section}

The other possibility is the direct scattering of WIMPs by electrons that are bound in atoms. The relevant Feynman diagram is depicted in Fig. 2 with quarks replaced by electrons. In this case only the electron flavor can be detected since the other flavors are not energetically allowed. Since the outgoing electrons are expected to have energies in the $\mathrm{eV}$ region one cannot ignore atomic binding effects. The binding energy $b$ is found from the tables of ionization potential (energy) of an atom 6

The problem is to find the cross section for WIMP scattered off an electron bounded in an atom. In order to proceed we shall make two simplifying assumptions :

1. As a working example, we shall assume that the target is a hydrogenic atom denoted by $H$ i.e., a nucleus with charge $+Z e$ and a single bounded electron with charge $-e$. We shall discuss deviations from this assumption throughout.

2. The gauge boson mediator X couples only to WIMP and leptons but not to quarks. This is a necessary condition to explain PAMELA positron excess of events. Therefore, this discussion refers strictly to model III in eq. (2.15) [see however footnote 2].

\footnotetext{
${ }^{6}$ Tables are normally given in $\mathrm{kJ} / \mathrm{mol}$, but they can easily be translated in $\mathrm{eV}$, since $96.485 \mathrm{~kJ} / \mathrm{mol}=1 \mathrm{eV}$. Thus for Cs we find $b=375.7 / 96.485=3.89 \mathrm{eV}$.
} 
There are four processes that could take place in WIMP + H-like atom collisions :

$$
\begin{aligned}
& \chi+H \quad \longrightarrow \quad+H \quad \text { (elastic) } \\
& \chi+H \quad \longrightarrow \quad \chi+H^{*} \quad \text { (inelastic) } \\
& \chi+H \quad \longrightarrow \quad \chi+e^{-}+H^{+} \quad \text { (production) }
\end{aligned}
$$

For the rest we shall consider only the situation (4.43). The elastic scattering (4.41) cannot be detected, and although we cannot exclude the inelastic one (4.42) from being experimentally probed through final state photons, we believe that it would be easier to detect the electrons from (4.43). We shall assume that the electron emerges with high momenta, $\mathbf{p}_{\mathbf{e}}^{\prime}$, such that in the final state its interaction with the Coulomb potential in $\mathrm{H}$-like atom is negligible, i.e, we can use plane wave states for incoming and outgoing particles. Using standard textbook [41] wavepacket analysis our starting point will be the cross section formula in the lab frame:

$$
\begin{aligned}
d \sigma & =\frac{1}{2 E_{\chi} 2 E_{e}} \frac{1}{|v|} \frac{d^{3} \mathbf{p}_{\chi}^{\prime}}{(2 \pi)^{3} 2 E_{\chi}^{\prime}} \frac{d^{3} \mathbf{p}_{\mathbf{e}}^{\prime}}{(2 \pi)^{3} 2 E_{e}^{\prime}}|\overline{\mathcal{M}}|^{2}(2 \pi) \delta\left(T_{\chi}-T_{\chi}^{\prime}-T_{e}^{\prime}-b\right) \\
& \times d^{3} \mathbf{p}_{\mathbf{e}}(2 \pi)^{3} \delta^{(3)}\left(\mathbf{p}_{\chi}+\mathbf{p}_{\mathbf{e}}-\mathbf{p}_{\chi}^{\prime}-\mathbf{p}_{\mathbf{e}}^{\prime}\right)\left|\phi\left(Z, \mathbf{p}_{\mathbf{e}}\right)\right|^{2},
\end{aligned}
$$

where $\mathbf{p}_{\chi}, \mathbf{p}_{\mathbf{e}}\left(\mathbf{p}_{\chi}^{\prime}, \mathbf{p}_{\mathbf{e}}^{\prime}\right)$ are the incoming (outgoing) three vector momenta of the WIMP and electron particles respectively, and $\overline{\mathcal{M}}$ is the matrix element of the process $\chi+e \rightarrow \chi+e$ averaged over the spins of the initial states calculated in Born approximation. We also ignore local velocity effects from the bound electron in the (static in lab frame) atom i.e., that is the relative velocity is $v \simeq v_{\chi}$. $T_{i}=p_{i}^{2} / 2 m_{i}, i=\chi, e$ are the kinetic energies and $b$ is the binding energy of the electron in H-atom $(\approx 13.6 \mathrm{eV})$. Moreover, in non-relativistic limit $E_{\chi} \simeq E_{\chi}^{\prime} \approx m_{\chi}$ and $E_{e} \simeq E_{e}^{\prime} \approx m_{e}$ with $m_{\chi} \gg m_{e}$, while $\phi_{n \ell m_{\ell}}(\mathbf{p})$, normalized at $\int_{V} d^{3} p\left|\phi_{n \ell m_{\ell}}(\mathbf{p})\right|^{2}=1$, is the Fourier transform of the coordinate wave function $\psi_{n \ell m_{\ell}}(\mathbf{r})$. Using the $\delta^{(3)}$-function to perform the integration over $\mathbf{p}_{\mathbf{e}}$, we obtain:

$$
d \sigma=\frac{|\overline{\mathcal{M}}|^{2}}{16 m_{\chi}^{2} m_{e}^{2} \beta} \frac{d^{3} \mathbf{p}_{\chi}^{\prime} d^{3} \mathbf{p}_{\mathbf{e}}^{\prime}}{(2 \pi)^{2}} \delta\left(\frac{\left|\mathbf{p}_{\chi}\right|^{2}}{2 m_{\chi}}-\frac{\left|\mathbf{p}_{\chi}^{\prime}\right|^{2}}{2 m_{\chi}}-\frac{\left|\mathbf{p}_{\mathbf{e}}^{\prime}\right|^{2}}{2 m_{e}}-b(Z)\right)\left|\phi_{n \ell m_{\ell}}\left(Z, \mathbf{p}_{\chi}^{\prime}+\mathbf{p}_{\mathbf{e}}^{\prime}-\mathbf{p}_{\chi}\right)\right|^{2},
$$

where the energy conservation delta-function has been written out explicitly. The result of eq. (4.45) is a product of two parts : a part that contains the dynamics of the WIMP-electron interaction through the matrix element $|\overline{\mathcal{M}}|$ times the probability of finding the target electron with momentum $\mathbf{p}_{\mathbf{e}}=\mathbf{p}_{\chi}^{\prime}+\mathbf{p}_{\mathbf{e}}^{\prime}-\mathbf{p}_{\chi}$ in H-atom. In addition the matrix element of the process $\chi+e \rightarrow \chi+e$ averaged over the spins of the initial states in Born approximation reads :

$$
|\overline{\mathcal{M}}|^{2} \simeq \frac{(16 \pi)^{2} \alpha_{\mathrm{DM}} \alpha^{\prime} m_{e}^{2} m_{\chi}^{2}}{\left(\left|\mathbf{p}_{\chi}-\mathbf{p}_{\chi}^{\prime}\right|^{2}-m_{X}^{2}\right)^{2}} s(\beta)
$$

where the factor $s(\beta) \equiv 1\left(\beta^{2}\right)$ for Dirac WIMP (Majorana WIMP) particle. Note that the cross section for Majorana WIMP is always smaller by a factor of $\beta^{-2}$ compared to the one involving Dirac WIMP. We now use the kinetic energy $\delta$-function appearing in eq. (4.45) in order to perform the $\left|\mathbf{p}_{\chi}^{\prime}\right|$ integration and arrive at:

$$
d \sigma=s(\beta) \frac{16 \pi^{2} \alpha_{\mathrm{DM}} \alpha^{\prime} m_{\chi}^{2}}{\left(\left|\mathbf{p}_{\chi}-\mathbf{p}_{\chi}^{\prime}\right|^{2}-m_{X}^{2}\right)^{2}} \frac{\left|\mathbf{p}_{\chi}^{\prime}\right|}{\left|\mathbf{p}_{\chi}\right|}\left|\mathbf{p}_{\mathbf{e}}^{\prime}\right|^{2} d\left|\mathbf{p}_{\mathbf{e}}^{\prime}\right|\left|\phi_{n \ell m_{\ell}}\left(Z, \mathbf{p}_{\chi}^{\prime}+\mathbf{p}_{\mathbf{e}}^{\prime}-\mathbf{p}_{\chi}\right)\right|^{2} d \xi d \eta,
$$


where the initial WIMP momentum is $\left|\mathbf{p}_{\chi}\right|=m_{\chi} \beta$ and the scattering angles are defined as

$$
\xi=\hat{p}_{\chi} \cdot \hat{p}_{\chi}^{\prime}, \quad \eta=\hat{p}_{\chi} \cdot \hat{p}_{e}^{\prime}, \quad \xi, \eta \in[-1,1] .
$$

The integration over the azimuthal angles has been carried out trivially in eq. (4.47) and the momentum $\left|\mathbf{p}_{\chi}^{\prime}\right|$ of the scattered WIMP is found to be

$$
\left|\mathbf{p}_{\chi}^{\prime}\right|=\sqrt{m_{\chi}^{2} \beta^{2}-2 m_{\chi} b(Z)-\frac{m_{\chi}}{m_{e}} p_{e}^{\prime 2}}, \quad \text { with } \quad p_{e}^{\prime}=\sqrt{2 m_{e} E_{e}^{\prime}},
$$

where $b(Z)$ is the ground state energy for hydrogenic atoms is

$$
b(Z)=\frac{Z^{2}}{2 a} \frac{e^{2}}{4 \pi}=\frac{Z^{2}}{2} m_{e} \alpha_{\mathrm{em}}^{2}, \quad a \simeq \frac{1}{m_{e} \alpha_{\mathrm{em}}},
$$

in the approximation $\mu \simeq m_{e}$ where $\mu$ is the reduced mass, with $\alpha_{\mathrm{em}}=\frac{e^{2}}{4 \pi} \approx 1 / 137, m_{e} \simeq 0.5$ $\mathrm{MeV}$ and $a=a_{0} \approx 0.5 \AA$ being the Bohr radius for $Z=1$. Throughout this chapter, we are going to use the ground state momentum distribution of hydrogenic atoms which reads:

$$
\phi_{100}(Z, p)=\frac{2^{3 / 2}}{\pi a} \frac{(Z a)^{5 / 2}}{\left(Z^{2}+p^{2} a^{2}\right)^{2}} .
$$

Notice that since $\phi_{100}(p)$ depends on $|p|^{2}$ and therefore from the scattering angles $\eta$ and $\xi$ and electron energy $E_{e}^{\prime}$. A term in eq. (4.47), $\frac{\left|\mathbf{p}_{\chi}^{\prime}\right|}{\left|\mathbf{p}_{\chi}\right|}=\frac{\left|\mathbf{v}_{\chi}^{\prime}\right|}{\left|\mathbf{v}_{\chi}\right|}$, arises from the fact that we treated the $\mathrm{H}$-atom as a brick wall potential. Had we not done so, the influence of the Coulomb potential on the emerging electron would not have been uniquely correlated to $\mathbf{p}_{\chi}^{\prime}, \mathbf{p}_{\chi}$ and the back reaction of the proton should have been taken into account.

Exactly the same result as in eq. (4.47) can be found by using simpler time-dependent perturbation theory for transitions to continuum in non-relativistic quantum mechanics [42]. In a more refined analysis however, when the recoiling energy is in the neighborhood of the binding energy of the atom one should take into account effects from the continuum hydrogenic wave functions instead of treating the final electron as plane wave. This analysis, though more accurate, is far more complicated and does not change the qualitative features of our results. It can be addressed in the future (together with other effects) especially if these kind of experiments become operative [see subsection 4.4 below].

We analyze below the corresponding cross sections for a massless and a massive mediator as we did in section 3 for the nucleons.

\subsubsection{Event Detection Rates}

In general for an atom, due to binding energy effects only the loosely bound electrons can contribute to the process (4.43). So we will convolute the elementary cross section with the WIMP velocity distribution, which, with respect to the galactic center, we will take to be Maxwell-Boltzmann form:

$$
f(\beta)=\left(\frac{3}{2<\beta^{2}>}\right)^{3 / 2} \frac{1}{\pi^{3 / 2}} e^{-\frac{3 \beta^{2}}{2<\beta^{2}>}} .
$$

Transforming this into the local coordinate system:

$$
\beta \rightarrow \beta \hat{\beta}+\beta_{0} \hat{z}=\beta \hat{\beta}+\sqrt{\frac{2<\beta^{2}>}{3}} \hat{z} \quad, \quad \beta^{2} \rightarrow \beta^{2}+\frac{2}{3}<\beta^{2}>+2 \beta \cos (\theta) \sqrt{\frac{2}{3}<\beta^{2}>},
$$


where $\theta$ is the angle between $\hat{\beta}$ and $\hat{z}$ and $\beta_{0}=\sqrt{\frac{2<\beta^{2}>}{3}}$ is the sun's velocity with respect to the center of the galaxy and $\left\langle\beta^{2}>\approx 10^{-6}\right.$. Then we obtain the local distribution of speeds $f_{\ell}(\beta)$ relative to the detector to be

$$
f_{\ell}(\beta)=\left(\frac{3}{2<\beta^{2}>}\right)^{3 / 2} \frac{1}{\pi^{3 / 2}} e^{-\left(\frac{3 \beta^{2}}{2<\beta^{2}>}+2 \beta \cos (\theta) \sqrt{\frac{3}{2<\beta^{2}>}}+1\right)} .
$$

The integration over the angles of the distribution can be done analytically. In evaluating the rate one has to incorporate the oncoming flux. So, adopting appropriate normalization, in the convolution we introduce the factor $1 / \sqrt{\left\langle\beta^{2}\right\rangle}$. This way we find the rate to be proportional to :

$$
\frac{\beta f_{\ell}(\beta) d \beta}{\sqrt{<\beta^{2}>}}=\left(\frac{3}{2<\beta^{2}>}\right)^{3 / 2} \frac{2}{\sqrt{\pi}} e^{-\left(\frac{3 \beta^{2}}{2<\beta^{2}>}+1\right)} \frac{\beta^{3}}{\sqrt{<\beta^{2}>}} \frac{\sinh \left(2 \beta \sqrt{3 /\left(2<\beta^{2}>\right)}\right)}{\beta \sqrt{3 /\left(2<\beta^{2}>\right)}} d \beta .
$$

Combining this with the cross section of eq. (4.47) obtained previously we arrive at:

$$
\left\langle\frac{d \sigma}{d E_{e}^{\prime}} \frac{\beta}{\sqrt{<\beta^{2}>}}\right\rangle=\int_{\beta_{\min }}^{\beta_{\mathrm{esc}}} d \beta \frac{\beta f_{\ell}(\beta)}{\sqrt{<\beta^{2}>}} \frac{d \sigma}{d E_{e}^{\prime}},
$$

where the lower velocity in the integral can be read from the positivity of the square root quantity in eq. (4.49)

$$
\beta_{\min }=\sqrt{\frac{2 E_{e}^{\prime}}{m_{\chi}}+\frac{2 b(Z)}{m_{\chi}}},
$$

and $\beta_{\mathrm{esc}}=2.84 \sqrt{(2 / 3)<\beta^{2}>}$ is the escape velocity. It is now easy to calculate the differential event rate per $\mathrm{eV}$ ejected electron energy per year and per kilogram of target material to be

$$
\frac{d R}{d E_{e}^{\prime}}=\frac{\rho_{0}}{m_{\chi}} \sqrt{<\beta^{2}>} N_{e}\left\langle\frac{d \sigma}{d E_{e}^{\prime}} \frac{\beta}{\sqrt{<\beta^{2}>}}\right\rangle,
$$

where $\rho_{0}=0.2 \mathrm{GeV} / \mathrm{cm}^{3}$ is the WIMP energy density and $N_{e}$ is the number of target electrons. Integration of eq. (4.58) upon $E_{e}^{\prime}$ over the region from $E_{e_{\min }}^{\prime}=0$ to $\left[m_{\chi} \beta_{\mathrm{esc}}^{2} / 2-b(Z)\right]$ results in the total event number per unit time and mass of the target which among other parameters depends on the mass and atomic numbers of the target atom. Moreover we shall display results on the total event rate $R(Z)$ when $E_{e_{m i n}}^{\prime}=E_{\text {th }}$ with varying experimental threshold energy $E_{\mathrm{th}}$.

\subsubsection{Time Modulation Effects for Electrons}

In the convolution of the elementary cross section we have so far considered only the motion of the sun with respect to the center of galaxy. More realistically, one should consider also the Earth's velocity and then find the modulated event rate that might be detected on Earth. In this case the WIMP velocity is read from

$$
\mathbf{v}^{\prime}=\mathbf{v}+v_{0} \hat{\mathbf{z}}+v_{1}(\sin \alpha \hat{\mathbf{x}}+\cos \alpha \cos \gamma \hat{\mathbf{y}}+\cos \alpha \sin \gamma \hat{\mathbf{z}}),
$$

where $v_{0}$ is Sun's velocity, $v_{1}$ is Earth's annual velocity, $\gamma=\frac{\pi}{6}$ is the angle between the projection of vector $\mathbf{v}_{\mathbf{1}}$ on the plane $y O z$ and the $\hat{\mathbf{y}}$ direction and $\alpha=a(t)$ is the complementary angle of the angle between $\mathbf{v}_{\mathbf{1}}$ and $\hat{\mathbf{x}}$. Then the WIMP cross section has to be convoluted with

$$
\left(\frac{\beta f_{\ell}(\beta) d \beta}{\sqrt{<\beta^{2}>}}\right)=\left(\frac{\beta f_{\ell}(\beta) d \beta}{\sqrt{<\beta^{2}>}}\right)_{0}(1+k \delta \cos \alpha),
$$


where the expression with the subscript " 0 " refers to eq. (4.55) with $\delta=\frac{v_{1}}{v_{0}} \approx 0.135$ and

$$
k=\left(2 \beta \sqrt{\frac{3}{2<\beta^{2}>}} \frac{\cosh \left(2 \beta \sqrt{\frac{3}{2<\beta^{2}>}}\right)}{\sinh \left(2 \beta \sqrt{\frac{3}{2<\beta^{2}>}}\right)}-3\right) \sin \gamma .
$$

It is now trivial to extend the distribution with energies event rate of eq. (4.58) with

$$
\frac{d R}{d E_{e}^{\prime}}=\left\langle\frac{d R}{d E_{e}^{\prime}}\right\rangle_{0}+\left\langle\frac{d R}{d E_{e}^{\prime}}\right\rangle_{\bmod } \times \cos \alpha
$$

where $\left\langle\frac{d R}{d E_{e}^{\prime}}\right\rangle_{0}$ is the unmodulated differential event rate while $\left\langle\frac{d R}{d E_{e}^{\prime}}\right\rangle_{\bmod }$ contains also the factor $k$ in eq. (4.61).

\subsection{Massless Mediator}

In this case dark matter scattering happens via the coupling of the exotic gauge boson to the photon (model II). The Feynman diagram is identical to the one presented in Fig. 2 2 with the quark replaced by electron. In general case the WIMP-electron cross section is not independent of the velocity. Thus, we will first estimate the cross section by using an average velocity $\sqrt{\left\langle\beta^{2}\right\rangle}=10^{-3}$. Following eq. (4.47) for a photonic mediator we find the differential cross section:

$$
\frac{d \sigma}{d E_{e}^{\prime}}=s(\beta) 16 \pi^{2} \alpha^{\prime} \alpha_{\mathrm{DM}} \kappa^{2} m_{\chi}^{2} m_{e} \frac{\left|\mathbf{p}_{\chi}^{\prime}\right|}{\left|\mathbf{p}_{\chi}\right|}\left|\mathbf{p}_{\mathbf{e}}^{\prime}\right| \int_{-1}^{1} d \xi \int_{-1}^{1} d \eta \frac{\left|\phi_{n \ell m_{\ell}}\left(Z, \mathbf{p}_{\chi}^{\prime}+\mathbf{p}_{\mathbf{e}}^{\prime}-\mathbf{p}_{\chi}\right)\right|^{2}}{\left(\mathbf{p}_{\chi}^{\prime}-\mathbf{p}_{\chi}\right)^{4}}
$$

where $\mathbf{q}=\mathbf{p}_{\chi}^{\prime}-\mathbf{p}_{\chi}$ is the WIMP momentum transfer which is $\xi$ dependent. The cross section peaks up the most from the forward direction $\xi \approx 1$. It should be mentioned that since the initial electron is bound, there is no infrared divergence in this case. Moreover, the momentum transfer can be as low as :

$$
|\mathbf{q}| \simeq 2 \frac{b(Z)+E_{e}^{\prime}}{\beta}
$$

This relation is important for explaining our numerical results below. Furthermore, in presenting the results we assume a Dirac WIMP fermion,i.e. $s(\beta)=1$. Furthermore, we choose a benchmark scenario inspired by our findings in nucleon decay :

$$
\beta=\sqrt{<\beta^{2}>}=10^{-3}, \quad Z=1, \quad \alpha_{\mathrm{DM}}=\alpha^{\prime}=\alpha_{\mathrm{em}}, \quad m_{\chi}=100 \mathrm{GeV}, \quad \kappa=10^{-10} .
$$

As it is obvious from eq. (4.63) it is very easy to apply our numerical results to any other parameters, $\beta, \alpha_{\mathrm{DM}}, \alpha^{\prime}, \kappa$, than those shown in eq. (4.65). We must note here that there is no parameter $\kappa$ in model III. This parameter is used here as a rescale factor and its very small value is adjusted so that we obtain rates of few events.

In Fig. [6a are shown the results for the $d \sigma / d E_{e}^{\prime}$ as a function of final electron's energy $E_{e}^{\prime}$ for three different cases of hydrogenic atoms with $Z=1, Z=3$ and $Z=6$ respectively. The differential cross section takes on its maximum values for final electron energy of around few $\mathrm{eV}$ for $Z=1$, around few tens of $\mathrm{eV}$ for $Z=3$ and around a hundred $\mathrm{eV}$ for $Z=6$. For the case $Z=1$, the extremum happens because of a fast increase of the term $\frac{\left|\mathbf{p}_{\chi}^{\prime}\right|}{\left|\mathbf{p}_{\chi}\right|}\left|\mathbf{p}_{\mathbf{e}}^{\prime}\right| \sim \sqrt{E_{e}^{\prime}}$ and the almost constant value of $\left|\phi_{100}\right|^{2}$ until $5 \mathrm{eV}$. For higher electron energies, e.g., $E_{e}^{\prime} \gtrsim 10 \mathrm{eV}$, the probability 

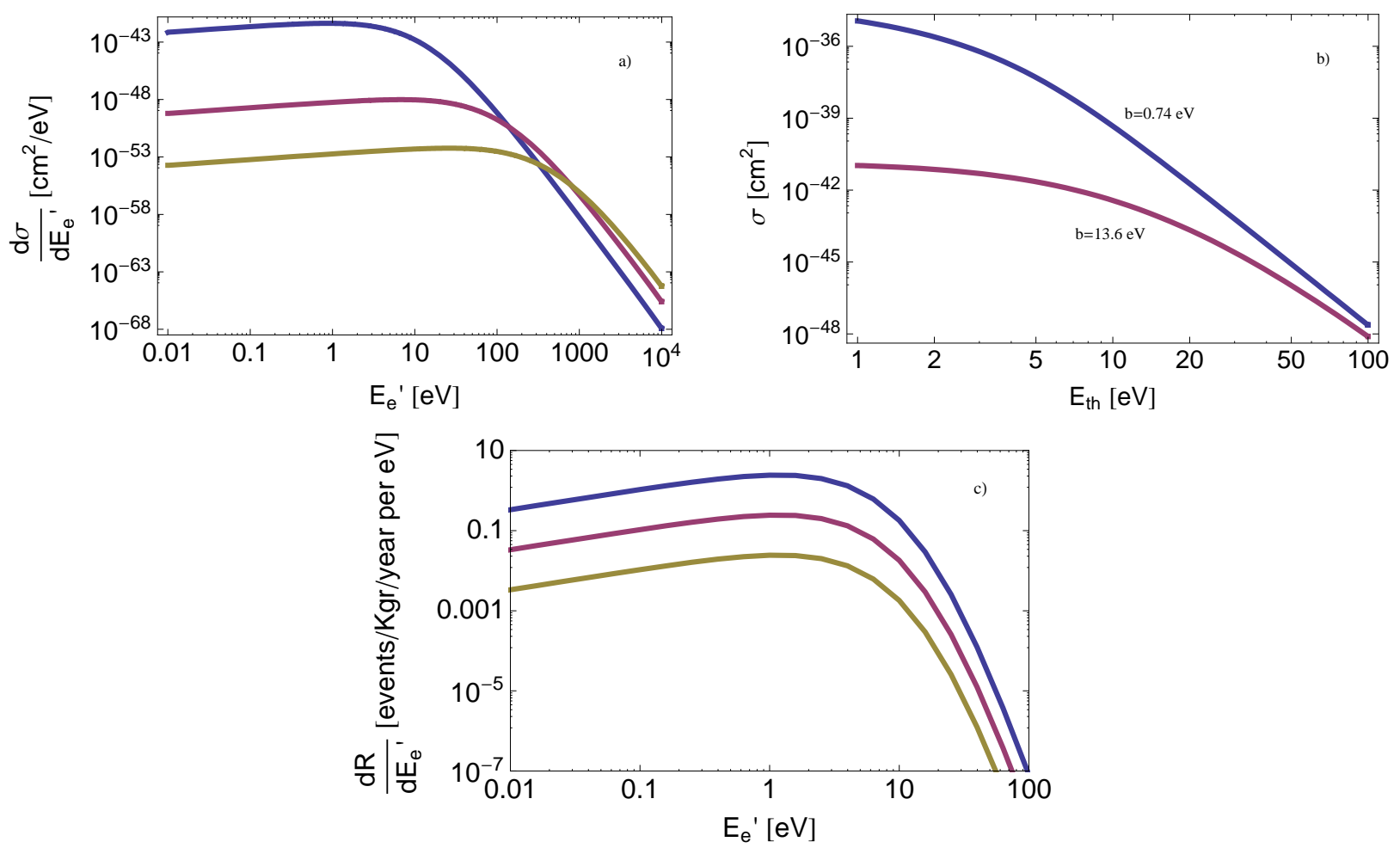

Figure 6: a) Predictions for $d \sigma / d E_{e}^{\prime}$ as a function of the ejected electron energy $E_{e}^{\prime}$. The target is assumed to be a hydrogenic atom in the ground state with $Z=1,3,6$ (from top to bottom). b) The total cross section for process (4.43) as a function of the experimental threshold energy for two binding energies. c) The differential event rate as a function of the electron energy and various WIMP masses $(10,100,1000) \mathrm{GeV}$ from (top to bottom). Other parameters not shown, are taken from eq. (4.65).

density factor $\left|\phi_{100}\right|^{2}$ drops fast as $1 / E_{e}^{\prime 8}$ and the term in the denominator of the integral increases as $E_{e}^{\prime 2}$, resulting in overall decreasing of the cross section as $E_{e}^{\prime-19 / 2}$. The same analysis can be used to describe the behavior of $d \sigma / d E_{e}^{\prime}$ in the other cases $(Z=3, Z=6)$. We must note here the in the limit $E_{e}^{\prime} \rightarrow 0$ we obtain $d \sigma / d E_{e}^{\prime} \rightarrow 0$ as the case should be. This is obscured in Fig. [6 due to the range choice of $E_{e}^{\prime}$.

Corresponding to the input parameters noted in (4.65) we calculate the total cross section from eq. (4.63) after numerical integration over $E_{e}^{\prime}$ in the region $\left[E_{\mathrm{th}}, m_{\chi} \beta^{2} / 2-b(Z)\right]$. Our results for $\sigma$ vs. the threshold energy $E_{\text {th }}$ are depicted in Fig. 6b. We have chosen two extreme cases of binding energies : $b=0.74 \mathrm{eV}$ that is the the binding energy of the electron bounded in the two electron atom $H^{-}$and $b=13.6 \mathrm{eV}$ that is the one corresponding to the $\mathrm{H}-$ atom we have been dealing so far. For $E_{\text {th }} \lesssim 10 \mathrm{eV}$ the difference in cross section is about three to six orders of magnitudes while for higher threshold energies becomes unimportant.

Following eq. (4.63) it turns out that the total cross section for process (4.43) is WIMP mass independent. It is experimentally useful to know how the cross section depends on the threshold energy $E_{\text {th }}$ that a given experiment can accomplish. This is plotted in Fig. 6 b. For $E_{\text {th }} \lesssim 1 \mathrm{eV}$, the cross section is essentially independent of $E_{\mathrm{th}}$. When the threshold becomes $5 \mathrm{eV}$, in the case of $b(Z)=13.6 \mathrm{eV}$, the cross section drops by a factor of $5 \mathrm{eV}$ while up to $10 \mathrm{eV}$ by a factor of 


\begin{tabular}{cccc}
\hline$E_{e}^{\prime}[\mathrm{eV}]$ & $\begin{array}{c}\left\langle\frac{d R}{d E_{e}^{\prime}}\right\rangle[\text { events } / \text { kgr } \\
\text { unmod. }\end{array}$ & $\begin{array}{c}\text { target/year } / \mathrm{eV}] \\
\text { mod. }\end{array}$ & $\mathrm{H}$ \\
\hline 0.1 & 0.11 & 0.01 & 0.09 \\
1 & 0.24 & 0.03 & 0.13 \\
10 & 0.02 & 0.002 & 0.10 \\
100 & $8.21 \times 10^{-9}$ & $1.04 \times 10^{-9}$ & 0.13 \\
\hline
\end{tabular}

Table 1: Time modulation effects in case of a photonic mediator following eq. (4.62) in the text. Various input parameters are given in eq. (4.65). $H$ is the ratio of the modulated divided by the unmodulated differential rate.

50. For smaller binding energy though, i.e., $b(Z)=0.74 \mathrm{eV}$, and up to $10 \mathrm{eV}$ the cross section decreases by three orders of magnitude.

Furthermore, the dependence of differential event rate $d R / d E_{e}^{\prime}$ as a function of the ejected electron energy $E_{e}^{\prime}$ for three different WIMP masses, $m_{\chi}=10,100,1000 \mathrm{GeV}$, is shown in Fig. 66. There is a maximum which follows the behavior of differential cross section. The event rate falls as $1 / m_{\chi}$ as the WIMP mass increases in accordance with eq. (4.58). For energy of few eV's and $m_{\chi}=10 \mathrm{GeV}$ we obtain a handful of events for $\kappa=10^{-10}$. A total event rate is obtained after integrating over the differential rate in Fig. 66. As a typical value, for $m_{\chi}=100 \mathrm{GeV}$ and the parameters in (4.65) we find $R\left(Z=1, \kappa=10^{-10}\right) \approx 1$ events/yr/target $\mathrm{kgr}$. The reader must recall here that this assumes a mixing parameter as small as $\kappa=10^{-10}$ !!

Finally, following the theoretical discussion of the previous subsection we examine effects of the WIMP time modulation. In Table 1 we display both the unmodulated and modulated differential event rate for four representative values of $E_{e}^{\prime}$ in the case of a massless mediator and parameters of eq. (4.65). The dimensionless parameter $H$, which is the ratio of the modulated by the non modulated differential amplitude, is constant around $9-13 \%$ independent of the energy and the WIMP mass. So the modulation $h=\delta \cdot k$ of the total rate is also going to be around $10 \%$, which means that the difference between the maximum (here always in June 3rd) and the minimum (here always in December) is $18-26 \%$, a result should not to be overlooked.

\subsection{Massive mediator}

Again the relevant diagram is the one of Fig. 2 with quarks replaced by electrons. By taking the non-relativistic limit of eq. (4.47) and the assumption that the momentum transfer in eq. (4.64) is much less than the mediator mass, $q^{2} \ll m_{X}^{2}$, we arrive at

$$
\frac{d \sigma}{d E_{e}^{\prime}}=s(\beta) \frac{16 \pi^{2} \alpha^{\prime} \alpha_{\mathrm{DM}} \kappa^{2}}{m_{X}^{4}} m_{\chi}^{2} m_{e} \frac{\left|\mathbf{p}_{\chi}^{\prime}\right|}{\left|\mathbf{p}_{\chi}\right|}\left|\mathbf{p}_{\mathbf{e}}^{\prime}\right| \int_{-1}^{1} d \xi \int_{-1}^{1} d \eta\left|\phi_{n \ell m_{\ell}}\left(Z, \mathbf{p}_{\chi}^{\prime}+\mathbf{p}_{\mathbf{e}}^{\prime}-\mathbf{p}_{\chi}\right)\right|^{2}
$$

In what follows we assume a Dirac WIMP fermion, i.e., $s(\beta)=1$. We assume the following input parameters :

$$
\begin{aligned}
\beta=\sqrt{<\beta^{2}>} & =10^{-3}, \quad Z=1, \quad \alpha_{\mathrm{DM}}=\alpha^{\prime}=\alpha_{\mathrm{em}} \\
m_{X} & =1 \mathrm{GeV}, \quad m_{\chi}=100 \mathrm{GeV}, \quad \kappa=1 .
\end{aligned}
$$

Although this parameter space violates the bounds in eqs. (2.16) and (2.17) it serves as a benchmark for this article in comparing results with those of section 3 if possible. The value of $\kappa$ is chosen 

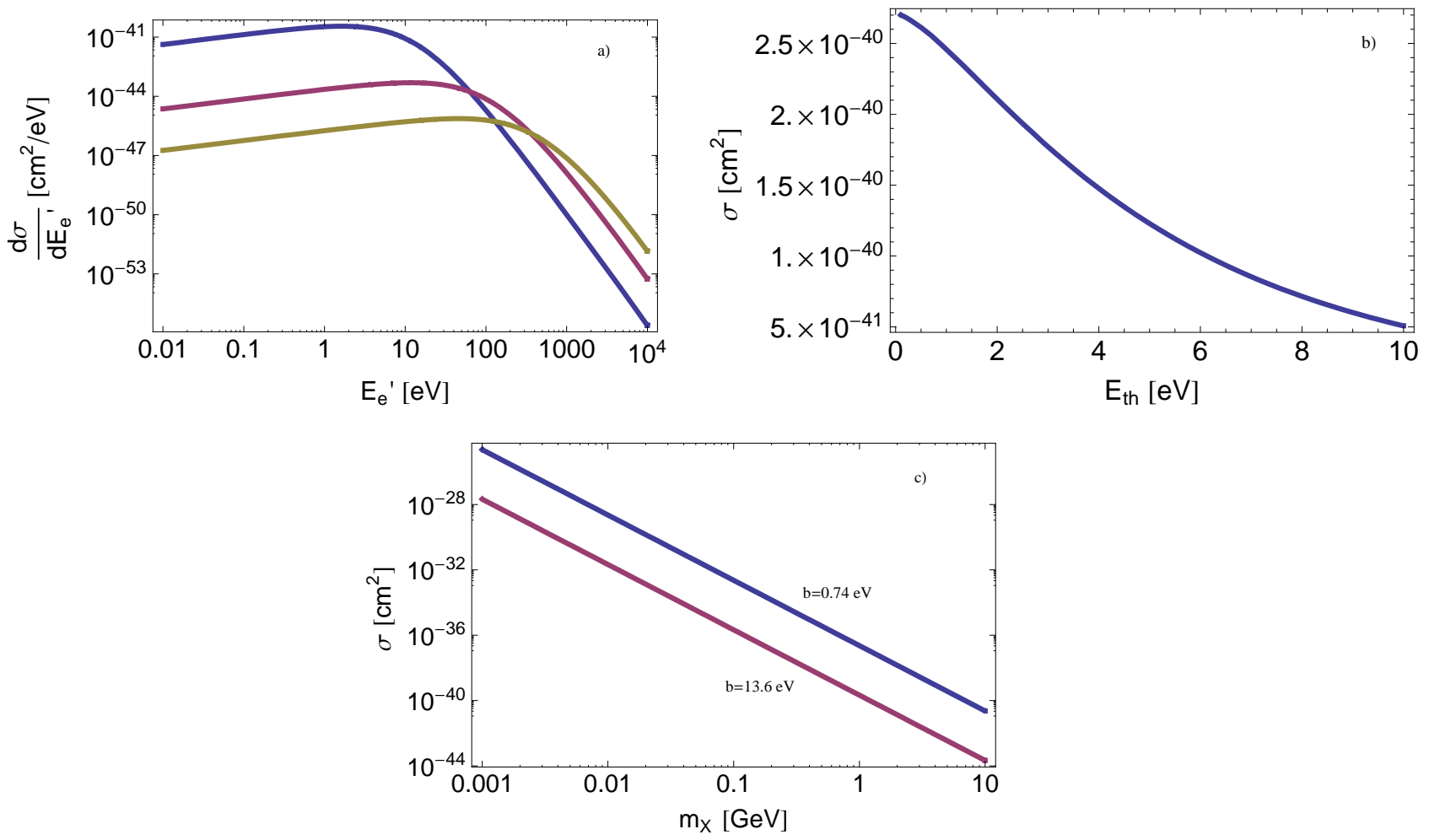

Figure 7: a) Predictions for $d \sigma / d E_{e}^{\prime}$ as a function of the ejected electron energy $E_{e}^{\prime}$. The target is assumed hydrogenic atom with $Z=1,3,6$ (from top to bottom) in the ground state. b) The total cross section as a function of threshold energy. c) The total cross section as a function of $m_{X}$ for two different binding energies. We assume a Dirac WIMP, $E_{\mathrm{th}}=0 \mathrm{eV}$ and input parameters from eq. (4.67) if not stated otherwise.

such that the resulting rate presented in the figures assumes no mixing of the $\mathrm{X}$-boson mediator which is formally the case of model III.

Results for the differential cross section $d \sigma / d E_{e}^{\prime}$ for the electron in the ground state of three hydrogenic atoms are shown in Fig. 7h. The differential cross section takes on its maximum values for final electron energy of around few $\mathrm{eV}$ for $Z=1$, ten of $\mathrm{eV}$ for $Z=3$ and around hundred $\mathrm{eV}$ for $Z=6$. For the case $Z=1$, the extremum happens because of a fast increase of the term $\frac{\left|\mathbf{p}_{\chi}^{\prime}\right|}{\mathbf{p}_{\chi} \mid}\left|\mathbf{p}_{\mathbf{e}}^{\prime}\right| \sim \sqrt{E_{e}^{\prime}}$ and the almost constant value of $\left|\phi_{100}\right|^{2}$ until $5 \mathrm{eV}$ [see eq. (4.66)]. For higher electron energies, e.g., $E_{e}^{\prime} \gtrsim 10 \mathrm{eV}$, the probability density factor $\left|\phi_{100}\right|^{2}$ drops fast as $1 / E_{e}^{\prime 8}$ resulting in overall decreasing of the cross section as $E_{e}^{\prime-15 / 2}$. In physical terms, the outgoing electrons of high energy demand high momenta in the initial electron wavefunction, which leads to suppression. The dependence on the $Z$ is easily explained if we recall that for hydrogenic atoms, $\left\langle p^{2}\right\rangle_{n=1}=Z^{2} p_{0}^{2}$ where $p_{0}$ is the Bohr momentum for Hydrogen. Furthermore, despite appearances in eq. (4.66), the differential cross section depends only very mildly on the WIMP mass. One can show analytically that the double integral over the wave function squared, is approximately proportional to $1 / m_{\chi}^{2}$ which cancels the $m_{\chi}^{2}$ in the numerator.

Corresponding to the input parameters noted in (4.67) we calculate the total cross section from eq. (4.66) after numerical integration over $E_{e}^{\prime}$ in the region $\left[E_{\mathrm{th}}, m_{\chi} \beta^{2} / 2-b(Z)\right]$. For fixed velocity, 

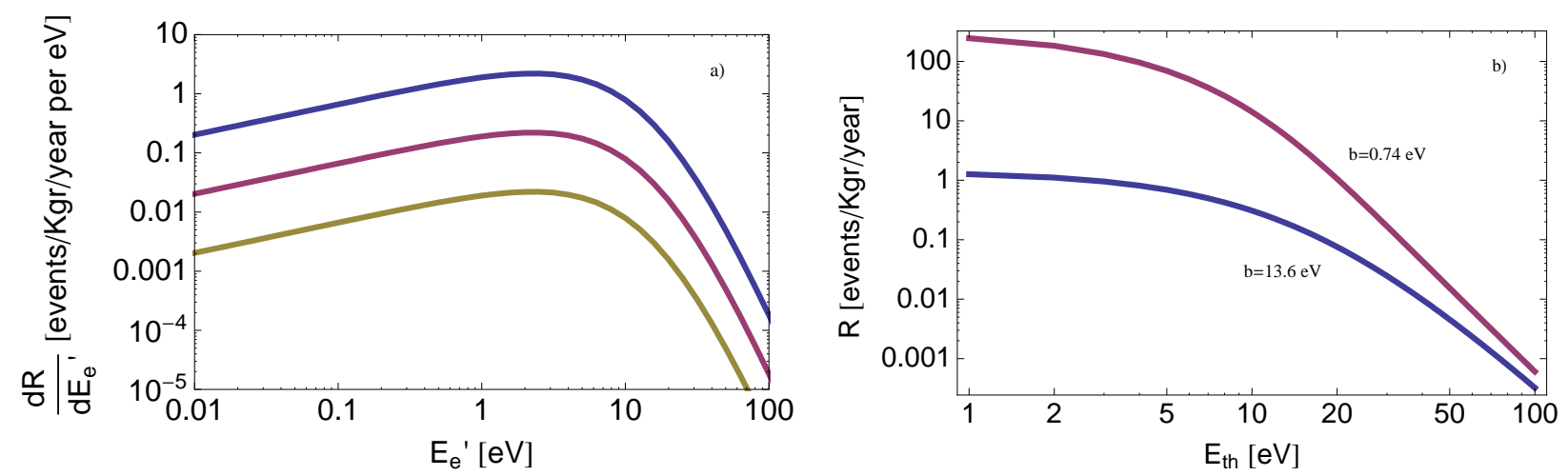

Figure 8: a) Differential event rate of Dirac WIMP scattered off hydrogen $(Z=1, A=1)$ target electrons per year per Kgr as a function of ejected electron energy $E_{e}^{\prime}$ in eV. Three different WIMP masses have been assumed : $m_{\chi}=10,100,1000 \mathrm{GeV}$, from top to bottom, respectively. b) The total event rate as a function of the experimental threshold energy for $m_{\chi}=100 \mathrm{GeV}$ for two different binding energies. Other input parameters are taken from eq. (4.67) for the massive mediator.

$\beta=0.001$, and $E_{\mathrm{th}}=0$ we find the following representative values :

\begin{tabular}{c|c}
$Z$ & $\sigma\left[\mathrm{cm}^{2}\right]$ \\
\hline 1 & $3 \times 10^{-40}$ \\
\hline 10 & $2 \times 10^{-44}$ \\
\hline 50 & $3 \times 10^{-48}$
\end{tabular}

The total cross section increases by a factor of about 32 when $\beta=\beta_{\text {esc }}$ is taken. The cross section decreases with $Z$ [see also Fig:7a], the reason being the fact that the binding energy increases with $Z^{2}$ [see eq. (4.50)] and therefore we need to go to larger - compared to ground state - momenta where the wavefunction is small despite their maximum value displacement towards larger momenta.

Assuming that the sensitivity of detecting low energy electrons will be analogous to the ongoing experiments $\left(\approx 10^{-43} \mathrm{~cm}^{2}\right)$, we could even extract bounds on various parameters in models I, II or III. From all running experiments, DAMA $[27,38]$ is the one that triggers on final state electrons with energy around $5 \mathrm{KeV}$. From Fig. 7h one obtains that, around that energy, the cross section is too small for $m_{X}=1 \mathrm{GeV}$ and all other inputs in eq. (4.67). However, $d \sigma / d E_{e}^{\prime} \propto m_{X}^{-4}$ and therefore for $m_{X} \approx 1 \mathrm{MeV}$ i.e., model types proposed in ref. [19], DAMA is a relevant experiment. Additionally, this is demonstrated in Fig. $7 \mathrm{k}$ where the total cross section as a function of $m_{X}$ is plotted for two reference values of binding energies.

In Fig. 7b we examine the total cross section as a function of the experimental energy threshold for low energies, relevant to our proposal. As we can see, the total cross section reduces by a factor of six in the region $0 \lesssim E_{\mathrm{th}} \lesssim 10 \mathrm{eV}$. Above $10 \mathrm{eV}$ the cross section drops drastically [see total rate in Fig. 8b].

Although not shown, we have also examined departures of the wavefunction from the ground state. The maximum value $d \sigma /\left.d E_{e}^{\prime}\right|_{\max }$ appears at the same place in $E_{e}^{\prime} \approx 1-10 \mathrm{eV}$. As an example, the difference in $d \sigma /\left.d E_{e}^{\prime}\right|_{\max }$ is an enhancement by a factor 20 when going from $1 s \rightarrow 2 s$. Furthermore, the size of the momentum transfer in conjunction with the non-zero binding energy are such that never let the wavefunctions to reach their zero nodes. 


\begin{tabular}{cccc}
\hline$E_{e}^{\prime}[\mathrm{eV}]$ & $\begin{array}{c}\left\langle\frac{d R}{d E_{e}^{\prime}}\right\rangle[\text { events/kgr } \\
\text { unmod. }\end{array}$ & $\begin{array}{c}\text { target/year } / \mathrm{eV}] \\
\text { mod. }\end{array}$ & $\mathrm{H}$ \\
\hline 0.1 & 0.06 & 0.01 & 0.17 \\
1 & 0.19 & 0.02 & 0.11 \\
10 & 0.079 & 0.008 & 0.10 \\
100 & $1.84 \times 10^{-5}$ & $1.78 \times 10^{-6}$ & 0.097 \\
\hline
\end{tabular}

Table 2: Time modulation effects in case of a massive mediator following eq. (4.62) and various input parameters in eq. (4.67). $H$ is the ratio of the modulated by the unmodulated differential amplitude.

Assuming one electron per target atom, and the average cross section of Fig. 7 a for $Z=1$, the differential event rate per eV of electrons energy per year per Kgr of hydrogen material as a functions of $E_{e}^{\prime}$ for various WIMP masses is depicted in Fig. 8 8 . The differential event rate again exhibits a maximum which follows that of the differential cross section calculated in Fig. 77. The event rate is of course higher for smaller WIMP mass [recall eq. (4.58)] and for electron energy of few eV's it varies from 0.01 up to 2 events $/ \mathrm{yr} / \mathrm{kgr} / \mathrm{eV}$ for $m_{\chi}=1000,10 \mathrm{GeV}$ respectively. For electron energy of around $100 \mathrm{eV}$ the role of the wave function is to reduce the differential rate by an order of magnitude i.e., from $10^{-4} \div 10^{-3}$ events $/ \mathrm{yr} / \mathrm{kgr} / \mathrm{eV}$. The total event rate for $m_{\chi}=100$ $\mathrm{GeV}$ and the other parameters in eq. (4.67) is predicted to be:

$$
R(Z=1, \kappa=1) \simeq 2[\text { events } / \text { yr } / \text { target } \mathrm{kgr}] .
$$

It is useful to know how the total rate (4.68) varies with an experimental threshold energy. This information can be extracted from Fig. $8 \mathrm{~b}$ for two different but judiciously chosen, values of binding energies. As in the case of the total cross section in Fig. $7 \mathrm{~b}$, the total rate drops by only a factor of five until $E_{\mathrm{th}} \approx 10 \mathrm{eV}$ while it drops very rapidly after about this scale. For example, it drops by a factor of $10^{4}$ for $E_{\text {th }}=100 \mathrm{eV}$. Smaller binding energies [upper line in Fig. 8b] result in up to two order of magnitude bigger rates but for threshold energies as low as $E_{\text {th }} \lesssim 5 \mathrm{eV}$.

Finally, in Table 2 we calculate the effects of time modulation and present the differential event rate for four different values of $E_{e}^{\prime}$ in the case of massive mediator with $m_{X}=1 \mathrm{GeV}$. We assume also a WIMP mass $m_{\chi}=100 \mathrm{GeV}$ and $Z=1$. The $H$ ratio is constant around $10 \%$ independent of the energy and the WIMP mass. So the modulation $h$ of the total rate is also going to be around $10-17 \%$, which means that the difference between the maximum (here always in June 3nd) and the minimum (here always in December) is $20-34 \%$.

\subsection{Experiment : The prospects of detecting single ultra low energy electrons}

As discussed in a previous section observation of light X- boson would require detectors with sub$\mathrm{keV}$ sensitivities. The development of such detectors, having a low energy threshold and low noise, remains generally a daunting challenge for present-day and future low- background experiments.

As shown in Fig. 6 the signal of low energy electrons produced by elastic collision process exhibits a maximum at energies around or even lower than $10 \mathrm{eV}$. At such energies a detector with single electron sensitivity will be required to reach a reasonable efficiency. The last ten years a particular effort is going on to develop ultra low threshold detectors in order to address low energy 
neutrino physics [43-46]. This has been achieved for low mass detectors. We are, however, seeking an even lower energy threshold.

Usual solid state detectors employed for dark matter projects have typical thresholds of a few $\mathrm{keV}$. It is very difficult to combine sub-keV and big mass at the same time. For instance UltraLow-Energy Germanium detectors [36] are able to reach a threshold of a few hundred eV's, but they are limited to a modular mass of a few grams. Anyway the achieved energy threshold is still below our requirements.

Single electron efficiency is achieved using detectors reaching very-high gains in order to cope with electronic noise. Gaseous detectors are good candidates. In such detectors high gains may easily be achieved. Having been conceived as a TPC Micromegas detector (MS) [47], it is compatible with large drift volumes and operation at high pressure, an example of which are the HELLAZ [48] prototypes. A great advantage of this detector is the versatility of target material: various gases from the lightest $\left(H_{2}\right)$ to heaviest $(\mathrm{Xe})$ could be used offering a large choice.

One idea to increase the mass of the target material is to use the recently developed Spherical Proportional Counter (SPC). This detector consists of large spherical gas volume with central electrode and radial electric field. Charges deposited in the drift volume are drifting to the central sensor where are amplified and collected. A novel concept of a proportional sensor, a metallic ball having a radius of about $15 \mathrm{~mm}$, located at the center of curvature, acting as a proportional amplification structure is used. It allows to reach high gas gains $(\geq 104)$ and operates from low to high gas pressure. At such gains, provided the low electronic noise of this detector, single electron efficiency is easily achieved.

The main advantages of the new structure relevant to our project are:

- Simplicity of the design.

- A single channel is used to read-out a large volume.

- Robustness

- The depth of the interaction, related to the rise time of the signal, is measured. This is important to apply fiducial cuts for background rejection purpose.

- Low detector capacity $\leq 0.1 \mathrm{pF}$, independent of the vessel size, allows very-low electronic noise, which is a key point toward achieving low energy threshold.

- Versatility of the target material and density; the detector is compatible with a large variety of gases and could operate from low pressure to high pressure. This could be a precious tool to identify a possible signal out of backgrounds.

A main concern of the proposed detection scheme is the minimal background level that will be reached by our system. By this one means that detector body and appropriate shield will be built with materials which are screened for low levels of natural and man-made radioactive impurities. Ordinary construction and shielding materials, however, do contain trace amounts of naturally occurring and man-made radionuclides which result in elevated background level; we need to design and fabricate the detector by careful material selection made out of low level activity.

Unfortunately, however, there exists very little experience at the very low energy (sub keV) region where our detector will be operating. An example is a low background gaseous detector with sub KeV energy threshold developed for solar axion search [50]; the reached background level 
is quite low and is flat in the sub $\mathrm{KeV}$ energy range down to $250 \mathrm{eV}$. Our purpose is to further decrease the energy threshold down to about $10 \mathrm{eV}$. This region has never been explored and therefore reaching the desired low level activity becomes a new experimental challenge. Single electron backgrounds could be emitted by materials pulled by the electric field through thermionic emission. The advantage of the spherical detector is that at the external vessel the electric field is extremely low and therefore highly reduced thermionic emission is expected.

The present prototype having a volume of $1 \mathrm{~m}^{3}$, filled with a gas at high pressure with a target mass of the order of $10 \mathrm{~kg}$ could fulfills sensitivity requirements for our project. We will search appropriate molecular gases having low binding energies and compatible with operation in the Spherical Proportional Counter detector [51].

At present it looks realistic to soon have a sphere of radius of 5 meters, which can be under a pressure of 5 bars. Thus, if one fills it with $80 \% \mathrm{Ar}$ and $20 \%$ Isobutane $\left(C_{4} H_{10}\right)$, one can have $212 \mathrm{Kg}$ of Hydrogen. With this much Hydrogen using eq. (4.68) and a threshold of $\approx 10 \mathrm{eV}$, we expect around 200 events per year for the parameters in (4.67). In models [19] where the mediator mass is very low, e.g. $m_{X} \approx 1 \mathrm{MeV}$, we expect an increase of the event rate by almost six orders of magnitude. Therefore, if a low energy experiment will be built it would possibly set the best limits on these kind of models.

\section{Conclusions}

Recent cosmic ray results from PAMELA, HESS and FERMI collaborations show an unexpected rising of positron events with energy that may be due to Dark Matter particle annihilations in the halo of our Galaxy. This Dark Matter particle "sees" the SM ones only through its interactions with an X-boson that couples to the SM gauge sector. Depending on the model, the mediator can be massless or massive with different couplings. In this article we study direct detection of this secluded type of dark matter employing nucleons or electrons with main emphasis in the latter case.

Due to the small momentum transfer 7 the massless case results in a large number of events that should have been seen by current nucleon recoiling direct detection experiments and therefore strong bounds on mixing parameters and couplings exist. Our work emphasizes the role of the low energy electron recoil in direct detection experiments and proposes a novel experimental avenue on how to proceed in searching for such low energy electrons. For simple hydrogenic atoms, and at low energy, $E_{e}^{\prime} \approx 10 \mathrm{eV}$, the cross section is enhanced by order of magnitudes compared to $\mathrm{KeV}$ recoil energies. In the neighborhood of low energies, the results depend highly on the binding energy of the ejected electron: the more loose the electron is the bigger the event rate becomes as expected. In this regard we considered two possibilities:

- The process is mediated by the massive mediator X (our model III).

In this case we do not have scattering off hadrons at tree level. So we do not have dominant constraints on the parameters of the model coming from the ongoing WIMP searches. Using the parameters of eq. (4.67) we have obtained fairly large cross sections for a Dirac WIMP. Employing the spherical TPC detector described above with a radius of $5 m$ under pressure of $5 \mathrm{Atm}$ we have found that we could have about 200 counts in a year, assuming a threshold of $10 \mathrm{eV}$. It is possible, however, that our choice of parameters is a bit optimistic and we may

\footnotetext{
${ }^{7}$ For nucleons, the momentum transfer is $\approx 2 \mathrm{MeV}$ and energy transfer is $\approx 2 \mathrm{KeV}$, while for low energy electron recoils they are $\approx 50 \mathrm{KeV}$ and $\approx 10 \mathrm{eV}$, respectively.
} 
have not considered all available constraints. Our results are also applicable to model-I. In this case however, due to the fact that couplings of the X-boson to hadrons appear at tree level, there exist strong constraints on the mixing parameter already from the nucleon direct searches [see eq. (3.38)] .

- The process is accommodated by the massless mediator (leptophylic version of model II) This mechanism is similar to that involving hadrons in section 3, one simply replaces the quarks by leptons. In this case we have found that the most stringent constraints on the parameters come from the standard WIMP searches. Thus using the parameters of Eq. (4.65) we have obtained with the above detector hundreds of events per year even with a (reduction) mixing coupling constant as low as $\kappa=10^{-10}$ for a Dirac WIMP. Such a huge signal cannot be seen by current experiments either due to lack of low energy threshold or because, experiments, like CDMS and XENON, are keeping only nuclear recoil events. We were surprised to find so large cross section. We now understand it, however, to be due to the photon propagator $\left(1 / q^{2}\right)^{2}$, which is favored by the fact that the momentum transfer is very low in the case of electrons. We should mention that, since the initial electron is bound, there is no infrared divergence and no need for a low energy cut off. It should be also noted that quark couplings to X-boson will come back through loop corrections even if they are forbidden at tree level by a symmetry which is eventually broken [see footnote 2]. Then current nucleon recoil experiments will be as important [see eq. (3.32)] and complementary to the electron ones.

The above conclusions assume that the WIMP is a Dirac particle. If the WIMP is Majorana particle the rates are suppressed by approximately a factor $\beta^{2} \approx 10^{-6}$. For both the above cases, annual time modulation effects are of the order of $20-30 \%$, important enough to be noticed.

We have limited the discussion of the rates in the case of hydrogen, since our cross section was evaluated using hydrogenic wave functions. Certainly the obtained rates will increase, if one can exploit the other atomic electrons with smaller binding energy. This situation was made manifest in our work with a judicious change of the binding energy [see Figs. 6b,7c,8b]. But then one should employ realistic wave functions.

In a similar fashion one can treat other dark matter candidates like right handed neutrinos, which arise in models in which the ordinary Dirac type mass is forbidden due to a discreet symmetry, but communication with the leptons is allowed via exotic scalars [52-54] with masses in the $50 \mathrm{GeV}$ region. It may also apply to other models involving exotic fermions and scalars recently proposed and reviewed in ref. [55].

\section{Acknowledgements}

A.D., and J.D.V., acknowledge partial support by the EU FP6 Marie Curie Research and Training Network "UniverseNet" (MRTN-CT-2006-035863). A.D. is also partially supported by the RTN European Programme, MRTN-CT-2006-035505 (HEPTOOLS, Tools and Precision Calculations for Physics Discoveries at Colliders). K.S. acknowledges full financial support from Greek State Scholarships Foundation (I.K.Y). 


\section{References}

[1] O. Adriani et al., arXiv:0810.4995 [astro-ph].

[2] O. Adriani et al., arXiv:0810.4994 [astro-ph].

[3] S.W. Barwick et al, Astrophys. J. 482 (1997) 1191; astro-ph/9703192.

[4] J.J. Beatty et al, Phys. Rev. Let. 93 (2004) 241102; astro-ph/0412230.

[5] M. Aguilar et al. [AMS-01 Collaboration], Phys. Lett. B $646 \quad$ (2007) 145 arXiv:astro-ph/0703154.

[6] A. A. Abdo et al. [The Fermi LAT Collaboration], arXiv:0905.0025 [astro-ph.HE].

[7] F. Aharonian et al. [H.E.S.S. Collaboration], Phys. Rev. Lett. 101 (2008) 261104 arXiv:0811.3894 [astro-ph]]; H. E. S. Aharonian, arXiv:0905.0105 [astro-ph.HE].

[8] M. Cirelli, M. Kadastik, M. Raidal and A. Strumia, Nucl. Phys. B 813, 1 (2009) arXiv:0809.2409 [hep-ph]].

[9] P. Meade, M. Papucci, A. Strumia and T. Volansky, arXiv:0905.0480 [hep-ph].

[10] J. Chang, et al, [ATIC Collaboration], Nature, 456, 362 (2008).

[11] D. P. Finkbeiner et al, Astrophys. J. 684 (2004) 186; astro-ph/0312547.

[12] D. Hooper, D. P. Finkbeiner and G. Dobler, Phys. Rev. D 76 (2007) arXiv 0705.3655.

[13] A.W. Strong et al, Astron. Astrophys. 444 (2005) 405; astro-ph/0509092.

[14] B. Holdom, Phys. Lett. B 166, 196 (1986); ibid. B 259329 (1991).

[15] M. Pospelov, A. Ritz and M. B. Voloshin, Phys. Lett. B 662, 53 (2008) arXiv:0711.4866 [hep-ph]]. Kinetic mixing of the photon with hidden $U(1) \mathrm{s}$ in string theory has been studied in S. A. Abel, M. D. Goodsell, J. Jaeckel, V. V. Khoze and A. Ringwald, JHEP 0807, 124 (2008) [arXiv:0803.1449 [hep-ph]].

[16] D. P. Finkbeiner and N. Weiner, Phys. Rev. D 76, 083519 (2007) arXiv:astro-ph/0702587.

[17] N. Arkani-Hamed, D. P. Finkbeiner, T. R. Slatyer and N. Weiner, Phys. Rev. D 79 (2009) 015014 arXiv:0810.0713 [hep-ph]]; N. Arkani-Hamed and N. Weiner, JHEP 0812 (2008) 104 arXiv:0810.0714 [hep-ph]]; M. Pospelov and A. Ritz, arXiv:0810.1502 [hep-ph].

[18] A. Sommerfeld, Ann. Phys. 11257 (1931);

Relevant to the abelian models considered here is the article, M. Cirelli, A. Strumia, M. Tamburini, Nucl. Phys. B 787 (2007) [arXiv:0706.4071 [hep-ph]].

[19] C. Boehm and P. Fayet, Nucl. Phys. B 683 (2004) 219 arXiv:hep-ph/0305261]; C. Boehm, P. Fayet and J. Silk, Phys. Rev. D 69, 101302 (2004) arXiv:hep-ph/0311143].

[20] E. C. G. Stueckelberg, Helv. Phys. Acta 11, 225 (1938).

[21] D. Feldman, Z. Liu and P. Nath, arXiv:0810.5762 [hep-ph]. 
[22] P. J. Fox and E. Poppitz, arXiv:0811.0399 [hep-ph].

[23] S. Baek and P. Ko, arXiv:0811.1646 [hep-ph].

[24] R. Harnik and G. D. Kribs, arXiv:0810.5557 [hep-ph].

[25] C. R. Chen, F. Takahashi and T. T. Yanagida, arXiv:0811.0477 [hep-ph].

[26] A. Ibarra, A. Ringwald, D. Tran and C. Weniger, JCAP 0908 (2009) 017 arXiv:0903.3625 [hep-ph]].

[27] R. Bernabei et al., Phys. Rev. D 77, 023506 (2008) [arXiv:0712.0562 [astro-ph]].

In the case of inelastic dark matter and DAMA prospects see, Y. Cui, D. E. Morrissey, D. Poland and L. Randall, JHEP 0905, 076 (2009) [arXiv:0901.0557 [hep-ph]].

[28] P. Q. Hung and J. J. Sakurai, Nucl. Phys. B 143 (1978) 81 [Erratum-ibid. B 148 (1979) 538].

[29] M. Baumgart, C. Cheung, J. T. Ruderman, L. T. Wang and I. Yavin, arXiv:0901.0283 [hep$\mathrm{ph}]$.

[30] For a recent review see, F. Jegerlehner and A. Nyffeler, arXiv:0902.3360 [hep-ph].

[31] M. Pospelov, arXiv:0811.1030 [hep-ph].

[32] D. E. Morrissey, D. Poland and K. M. Zurek, arXiv:0904.2567 [hep-ph].

[33] J. D. Bjorken, R. Essig, P. Schuster and N. Toro, arXiv:0906.0580 [hep-ph].

[34] B. Batell, M. Pospelov and A. Ritz, arXiv:0906.5614 [hep-ph].

[35] B. Kors and P. Nath, Phys. Lett. B 586 (2004) 366 arXiv:hep-ph/0402047]; D. Feldman, Z. Liu and P. Nath, Phys. Rev. D 75 (2007) 115001 arXiv:hep-ph/0702123.

[36] J. Yoo [CDMS Collaboration], arXiv:0810.3527 [hep-ex].

[37] J. Angle et al. [XENON Collaboration], Phys. Rev. Lett. 100 (2008) 021303 arXiv:0706.0039 [astro-ph]].

[38] R. Bernabei et al. [DAMA Collaboration], Eur. Phys. J. C 56, 333 (2008) arXiv:0804.2741 [astro-ph]].

[39] J. D. Vergados, J. Phys. G 30, 1127 (2004) arXiv:hep-ph/0406134.

[40] N.Tetradis, J.D. Vergados and A. Faessler, Phys. Rev. D 75, 023504 (2007)

[41] See for instance, M. E. Peskin and D. V. Schroeder, "An Introduction To Quantum Field Theory," Reading, USA: Addison-Wesley (1995) $842 p$.

[42] See for example,

Hans A. Bethe, "Intermediate Quantum Mechanics," Lecture notes and supplements in physics. Notes by R. W. Jackiw.

L. Schiff, "Quantum Mechanics," McGraw-Hill Education (1968) $584 p$.

[43] J. I Collar, I. Giomataris, Nucl. Instrum. Meth. A471:254-259,2000. 
[44] I. Giomataris, J, D. Vergados, Nucl. Instrum. Meth. A530:330-358,2004.

[45] Chris Hagmann, Adam Bernstein, IEEE Trans. Nucl. Sci. 51:2151-2155,2004.

[46] Henry T. Wong, Mod. Phys. Lett. A23:1431-1442,2008.

[47] Y. Giomataris, P. Rebourgeard, J. P. Robert and G. Charpak, Nucl. Instrum. Meth. A 376 (1996) 29.

[48] P. Gorodetzky et al., Nucl. Instrum. Meth. A 433, 554 (1999).

[49] P. Buzhan et al., Nucl. Instrum. Meth. A 504, 48 (2003).

[50] P. Abbon et al., New J. Phys. 9 (2007) 170 arXiv:physics/0702190].

[51] I. Giomataris et al., JINST 3, P09007 (2008) [arXiv:0807.2802 [physics.ins-det]].

[52] R. Adhikari, J. Erler and E. Ma, Phys. Lett. B 672 (2009) 136 [arXiv:0810.5547 [hep-ph]].

[53] Q. H. Cao, E. Ma and G. Shaughnessy, arXiv:0901.1334 [hep-ph].

[54] D. Suematsu, T. Toma and T. Yoshida, arXiv:0903.0287 [hep-ph].

[55] E. Ma, arXiv:0810.5574 [hep-ph]. 